\title{
ALPHA AND "Be CLUSTER AMPLITUDES AND CORE EXCITATIONS IN s-d SHELL NUCLEI
}

\author{
K. T. HECHT ${ }^{\dagger}$ \\ David M. Dennison Bullding, University of Michigan, Ann Arbor, Michtgan 48109, USA
}

Received 2 November 1976

\begin{abstract}
Abetract: SU(3) and SU(4) recoupling are used to develop techniques for the calculation of norm and overlap matrix elements for properly antisymmetrized cluster wave functions. These techniques are illustrated in detail by the calculation of $\alpha$ and ${ }^{8} \mathrm{Be}$ cluster amplitudes for states of a few hw excitation in s-d shell nuclei. These extend carlier SU(3) strong coupling results for states of highest intrinsic deformation to all values of $(\lambda \mu)$, making it possible to calculate $\alpha$-amplitudes for SU(3) weak and intermediate coupling wave functions. The extreme SU(3) weak and strong coupling limits for $\alpha$-amplitudes are compared for states of 1 how excitation in light a-d shell nuclei. A general formulation is given for the calculation of spectroscopic amplitudes for the transfer of ${ }^{8} \mathrm{Be}$ clusters in $\left({ }^{12} \mathrm{C}, \alpha\right)$ or $\left({ }^{14} \mathrm{~N},{ }^{6} \mathrm{Li}\right)$ reactions on nuclei for which SU(3) and SU(4) are meaningful symmetries. The method is illustrated by predictions of ${ }^{\circ} \mathrm{Be}$ amplitudes to the prominent rotational bands in ${ }^{20} \mathrm{Ne}$.
\end{abstract}

\section{Introduction}

Recent multinucleon transfer reactions induced by ${ }^{6} \mathrm{Li}$ or heavy ion projectiles have furnished much new information on cluster structures in nuclei over a wide mass range ${ }^{1}$ ). The relationship between cluster spectroscopic amplitudes and the norm and overlap kernels of properly antisymmetrized resonating group wave functions ${ }^{2-4}$ ) is a very useful one since much progress has recently been made in the treatment of nuclear systems by the resonating group method. The most elegant techniques for the calculation of resonating group kernels make use of various integral trąnsforms ${ }^{5-9}$ ). Although many challenging nuclear problems have been investigated by this technique ${ }^{10-13}$ ), applications have been limited mainly to very light nuclei $(A \leqq 12)$. Closely related techniques making use of harmonic oscillator generating functions have been developed by a group of Japanese workers ${ }^{14-18}$ ). Their method is particularly simple if the internal and relative motion wave functions of the resonating groups are constructed from harmonic oscillator wave functions with the same oscillator size parameter. Much of the power of the technique comes from the recognition ${ }^{16}$ ) that the eigenfunctions of the overlap operator, $K$, are SU(3) strongcoupled cluster wave functions. For this reason, however, many of the applications with this technique, particularly in the approximation of the so-called orthogonality

' Supported by the US National Science Foundation. 
condition model ${ }^{19}$ ), have been limited to nuclei near the doubly magic system ${ }^{16} \mathrm{O}$, with $\mathrm{SU}(3)$ symmetry $(\lambda \mu)=(00)$. Specific applications include for example, $\alpha+{ }^{12} \mathrm{C}$ [ref. $\left.{ }^{20}\right)$ ], $\alpha+{ }^{16} \mathrm{O}$ [refs. $\left.{ }^{21-24}\right)$ ], $3 \alpha$ [refs. $\left.{ }^{17,25}\right)$ ] and $2 \alpha+{ }^{16} \mathrm{O}$ [ref. $\left.{ }^{17}\right)$ ]. Supermultiplet symmetry and harmonic oscillator properties have been elegantly exploited by Kramer and collaborators ${ }^{26-28}$ ) in their studies of nucleon clustering in light nuclei. Both SU(3) and SU(4) symmetry properties have also been exploited by Smirnov et al. ${ }^{29,30}$ ) in terms of a translationally invariant shell-model description, but applications again have been restricted mainly to very light nuclei.

It is the purpose of the present work to show that the calculation of cluster spectroscopic amplitudes can be extended, for states up to a few hw of oscillator excitation, to any nuclear system for which $S U(3)$ is a meaningful symmetry, hence for nuclei through much of the s-d shell and certain core-excited states of nuclei reaching up into the $\mathrm{Ca}$ region. By using harmonic oscillator SU(3) strong-coupled resonating group wave functions the calculation of cluster spectroscopic amplitudes is reduced to an exercise in SU(3) and SU(4) recoupling and is made feasible by the simplicity and ready availability of the needed SU(3) and SU(4) recoupling coefficients ${ }^{31-33}$ ), as well as the needed shell-model parentage coefficients in the SU(3)-SU(4) scheme ${ }^{34}$ ). The complications of embedding the angular momentum in the SU(3) symmetry are saved till the last step in any calculation. This step is again made feasible through the ready availability of a computer code for the Wigner coefficients in the $\mathrm{SU}(3) \supset \mathbf{R}(3)$ basis ${ }^{32}$ ).

Although the techniques presented here can be used to calculate spectroscopic amplitudes for any few-nucleon cluster, the applications in this work will be limited to $\alpha$-clusters and ${ }^{8} \mathrm{Be}$ (or double $\alpha$ ) clusters. Since the internal wave functions for these clusters can be approximated by SU(4) scalar functions, the SU(4) recoupling for these systems is essentially trivial. Alpha spectroscopic amplitudes for s-d shell nuclei and states of $0 \hbar \omega$ excitation have been calculated by Draayer ${ }^{35}$ ) using the c.f.p. of ref. ${ }^{34}$ ). Predictions based on the simplest SU(3) approximations seem to be in remarkably good agreement with experiment ${ }^{36-39}$ ) and differ but little from those calculated with much more complicated shell-model wave functions ${ }^{40}$ ). Some $\alpha$-spectroscopic amplitudes for states of $1 \hbar \omega$ and $2 \hbar \omega$ excitation in s-d shell nuclei have been given in ref. ${ }^{34}$ ); but these results are limited to cluster states which can be approximated by SU(3) strong-coupled wave functions, with SU(3) quantum numbers which in general are high enough so that the states are automatically free of spurious c.m. excitation. There is some evidence ${ }^{20.41-42}$ ) that a weak coupling approximation may be better for states of a few $\hbar \omega$ excitation in s-d shell nuclei. Alpha spectroscopic amplitudes in the weak coupling approximation can be calculated once the strong coupling amplitudes for translationally invariant cluster states have been calculated for states of all possible SU(3) couplings of the internal and relative motion functions by the technique to be presented here.

Sect. 2 establishes the notation and outlines the general method of calculation. Calculations of $\alpha$-cluster amplitudes are illustrated in sect. 3. Detailed results are 
given for states of $1 \hbar \omega$ excitation in nuclei in the beginning of the s-d shell. Results of the extreme weak and strong $\mathrm{SU}(3)$ coupling approximations are compared, and tabulations are given of the SU(3) norm factors needed to calculate $\alpha$-spectroscopic amplitudes for any intermediate coupling wave function. Since the extraction of $\alpha$-spectroscopic amplitudes from direct reaction cross sections or decay widths must make use of realistic $\alpha$-residual nucleus relative motion wave functions, harmonic oscillator $\alpha$-spectroscopic amplitudes may be needed up to many units of $h \omega$ oscillator excitation. As a further example of the present technique SU(3) norm factors are therefore given for the ${ }^{24} \mathrm{Mg}=\alpha+{ }^{20} \mathrm{Ne}$ system for all states based on a $(\lambda \mu)=(80)$ ${ }^{20} \mathrm{Ne}$ core up to oscillator excitations of $4 h \omega$. Sect. 4 illustrates the technique with a more complicated cluster, the ${ }^{8} \mathrm{Be}$ (or double $\alpha$ ) cluster. Calculations of ${ }^{8} \mathrm{Be}$ spectroscopic amplitudes for s- $d$ shell nuclei have so far been limited to the nucleus ${ }^{24} \mathrm{Mg}$. This has been treated by Katō and Bandō ${ }^{17}$ ) who have calculated ti.e eigenvalues of the overlap operator, $K$, for the ${ }^{16} \mathrm{O}+\alpha+\alpha$ system by an elegant generating function technique which, however, is tailored to a core of SU(3) symmetry $(\lambda \mu)=(00)$. Interesting rotational bands are known in ${ }^{20} \mathrm{Ne}$ and ${ }^{40} \mathrm{Ca}$ which can be reached only by eight-nucleon transfer but not by four-nucleon transfer reactions [refs. $\left.\left.{ }^{43-48}\right)\right]$. Spectroscopic amplitudes for core +8 -particle systems with a core of arbitrary SU(3) symmetry are therefore of interest. Specific applications are given of ${ }^{8} \mathrm{Be}$ spectroscopic amplitudes to certain core-excited rotational bands in ${ }^{20} \mathrm{Ne}$.

\section{Method of calculation}

The general method of calculation will be illustrated with the simplest example, a single $\alpha$-cluster coupled to a core residual nucleus. The cluster wave function for an $\alpha+$ core-system can be specified in SU(3) strong-coupled form by

$$
\Psi^{\prime \prime}=\mathscr{A}\left[\phi_{A-4}\left(\xi_{c}\right)^{\left(\lambda_{c} \mu_{c}\right)\left[f_{c]}\right]} \times \phi_{4}\left(\xi_{\alpha}\right) \Phi^{(20)}\left(r_{c-\alpha}^{\prime}\right)\right]_{x L S J M J T M T}^{\left(\lambda_{\mu}\right)\left(\gamma_{M}^{\prime}\right)}
$$

where the internal wave functions, $\phi(\xi)$, are functions of intrinsic coordinates $\xi$, and hence free of spurious c.m. excitation. It is assumed that the internal functions $\phi_{A-4}$ and $\phi_{4}$ are constructed from single particle harmonic oscillator wave functions $\Phi\left(r_{i}^{\prime}\right)$ with $r_{i}^{\prime}=[\mathrm{m \omega} / \hbar]^{\frac{1}{t}} \boldsymbol{r}_{i}$, while the wave function for the relative motion of the cluster, $\Phi\left(r_{c-\alpha}^{\prime}\right)$, is also a harmonic oscillator wave function. The prime on the variable $r$ again indicates a dimensionless coordinate, which is now the relative coordinate $r_{c-a}^{\prime}=[4(A-4) m \omega / A \hbar]^{t} r_{c-\infty}$ The harmonic oscillator frequency $\omega$ is assumed to be the same in all size parameters. It is also assumed that the core cluster function has good SU(3) and supermultiplet symmetry. The SU(3) quantum numbers are given by the Elliott labels $(\lambda \mu)$, the SU(4) quantum numbers by [f] derived from $\mathrm{U}(4)$ partition numbers conjugate to the space symmetry labels $[f]$. [The notation follows that of refs. ${ }^{31,33,34}$ ). The $\alpha$-particle internal function is built from 0 s oscillator functions and transforms according to scalar representations of SU(3) and SU(4). [The quantum numbers $\left(\lambda_{a} \mu_{a}\right)=(00),\left[f_{z}\right]=[0]$ are omitted but are 
to be quietly understood.] In this strong-coupled form the square bracket of eq. (1) designates SU(3) and SU(4) coupling (the latter being trivial with $\left[f_{a}\right]=[0]$ ). The SU(3) strong-coupled cluster function can thus be expanded in angular momentum coupled functions :

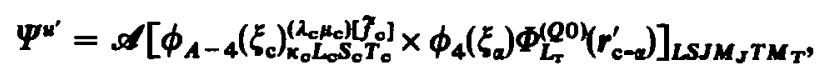

where the square bracket now designates ordinary angular momentum coupling in the separate orbital, spin, and isospin spaces (the latter being trivial with $S_{\alpha}=0, T_{\alpha}=0$ ). The SU(3) strong-coupled functions are related to these angular momentum coupled functions by

$$
\begin{aligned}
& \mathscr{A}\left[\phi_{A-4}\left(\xi_{c}\right)^{\left(\lambda_{c} \mu_{c}\right)\left[f_{01}\right]} \times \phi_{4}\left(\xi_{\alpha}\right) \Phi^{(00)}\left(r_{c-\alpha}^{\prime}\right)\right]_{\kappa L L J M J T M_{T}}^{(\lambda \mu)\left(\gamma_{J}\right.}
\end{aligned}
$$

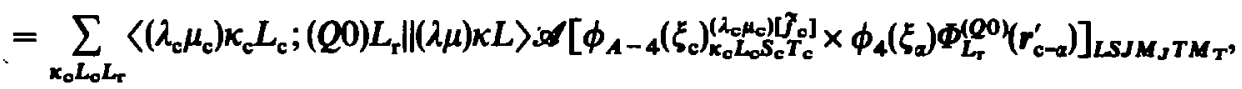

where the double-barred coefficients are reduced $S U(3) \supset \mathbf{R}(3)$ Wigner coefficients in the notation of Draayer and Akiyama ${ }^{31}$ ). [Subgroup labels and phase conventions are those of ref. ${ }^{31}$ ). ] In the special case $S_{c}=0$ the cluster function (2a) corresponds to an SU(3) weak-coupled wave function in which only the internal cluster wave functions and the relative motion wave functions have good SU(3) quantum numbers. With $S_{c} \neq 0$, a cluster wave function in the $\mathrm{SU}(3)$ weak coupling approximation must correspond to a definite state of the core cluster. It should have good $J_{c}$, and $\kappa_{J_{\mathrm{c}}}$ if it is the band head of a rotational band of good $\kappa_{J_{c}}\left[\right.$ refs. ${ }^{31,35}$ )] (rather than the $\kappa_{\mathrm{c}}$ associated with the orbital angular momentum $L_{\mathrm{c}}$ ). In this case an SU(3) weak-coupled cluster function has the form

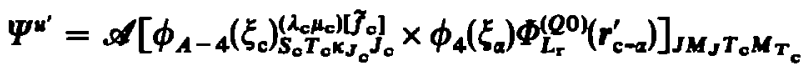

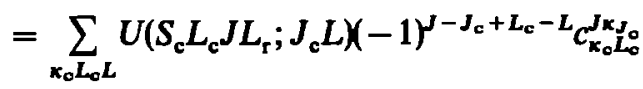

$$
\begin{aligned}
& \times \mathscr{A}\left[\phi_{A-4}\left(\xi_{c}\right)_{x_{c} L_{c} S_{c} T_{c}}^{\left(\lambda_{q} \mu_{c}\right)} \times \phi_{4}\left(\xi_{q}\right) \Phi_{L_{r}}^{(Q O)}\left(r_{c-\alpha}^{\prime}\right)\right]_{L S_{d} J M_{J} T_{c} M_{T_{c}}} .
\end{aligned}
$$

In this SU(3) weak-coupled function the angular momentum coupling is that of $J_{c}$ with $L_{\mathrm{r}}$ to total $J$. The $U$-coefficient is an angular momentum recoupling (Racah) coefficient in unitary form which relates this function to the function defined in eq. (2a). The $c_{\kappa_{\mathrm{o}} L_{\mathrm{o}}}$ are expansion coefficients of states with good $\kappa_{J_{\mathrm{c}}} J_{\mathrm{c}}$ in terms of states of the $\kappa_{\mathrm{c}} L_{\mathrm{c}}$ basis. The antisymmetrizer $\mathscr{A}$ of eqs. (1) and (2) exchanges only nucleons between different clusters. It is normalized according to

$$
\mathscr{A}=\left[\left(\begin{array}{l}
A \\
4
\end{array}\right)\right]^{-t}\left[1+\sum_{k}(-1)^{k} P_{k}\right]
$$

where the $P_{k}$ are permutation operators which exchange at least one pair of nucleons from the $\alpha$ and $(A-4)$ particle clusters. 
The overlap between two cluster wave functions of the type (1) can be expressed in terms of a Feshbach overlap operator ${ }^{49}$ ), $K$,

$$
\left\langle\Psi^{v} \mid \Psi^{u}\right\rangle=\langle v|1-K| u\rangle,
$$

where $|\Psi \eta=\mathscr{A}| u\rangle$ and $u$ stands for the full set of quantum numbers, $\left(\lambda_{c} \mu_{c}\right)$ $\left[f_{c}\right](Q 0)(\lambda \mu) \kappa L .$. , of wave function (1). The diagonal matrix elements of the operator, $1-K$, serve to normalize the antisymmetrized cluster functions. Pauliforbidden or redundant states are eigenstates of $1-K$ with eigenvalue zero. The simplicity of the harmonic oscillator SU(3) strong-coupling basis was exploited by Horiuchi ${ }^{16}$ ) through the recognition that the $K$-operator is an SU(3) scalar. The $K$-operator is thus diagonal in $(\lambda \mu)$, and its matrix elements are independent of the subgroup labels $\kappa L \ldots$ of eq. (1). The overlaps of functions of type (1) are diagonal in $(\lambda \mu)$ and independent of $\kappa L \ldots$. In the single channel approximation of the SU(3) strong-coupling scheme (based on a core state with a single $\left.\left(\lambda_{c} \mu_{c}\right)\left[f_{c}\right]\right)$ the operator $1-K$ is thus a simple number which gives the normalization of the cluster function (1). In this case $1-K=1 / N^{2}$, where the normalized cluster function is $N \Psi^{\prime \prime}$, and $N$ is dependent on $\left(\lambda_{\mathrm{c}} \mu_{\mathrm{c}}\right),\left[\widetilde{f}_{\mathrm{c}}\right], Q,(\lambda \mu)$ but independent of $\kappa L \ldots$, and is abbreviated by $N_{(\lambda \mu)}^{Q}$. The normalization constant of the corresponding weakcoupling wave function (2) is abbreviated by $N_{L_{r}}^{Q}$. With $S_{c}=0$ it is related to the $N_{(\lambda \mu)}^{Q}$ by

$$
\frac{1}{\left[N_{L_{r}}^{Q}\right]^{2}}=\sum_{(\lambda \mu)} \frac{1}{\left[N_{(\lambda \mu)}^{Q}\right]^{2}} \sum_{\kappa}\left\langle\left(\lambda_{c} \mu_{c}\right) \kappa_{c} L_{c} ;(Q 0) L_{r} \|(\lambda \mu) \kappa L\right\rangle^{2}
$$

With $S_{c} \neq 0$, the normalization constant of the SU(3) weak-coupled function (2b) is given by

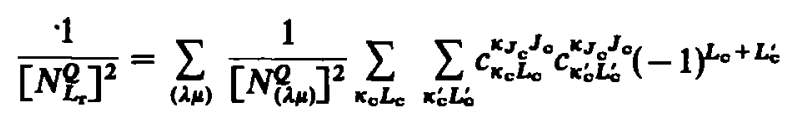

$$
\begin{aligned}
& \times \sum_{\mathbf{L}} U\left(S_{\mathrm{c}} L_{\mathrm{c}} J L_{\mathrm{r}} ; J_{\mathrm{c}} L\right) U\left(S_{\mathrm{c}} L_{\mathrm{c}} J L_{\mathrm{r}} ; J_{\mathrm{c}} L\right) \\
& \times \sum_{\kappa}\left\langle\left(\lambda_{\mathrm{c}} \mu_{\mathrm{c}}\right) \kappa_{\mathrm{c}} L_{\mathrm{c}} ;(Q 0) L_{\mathrm{r}} \|(\lambda \mu) \kappa L\right\rangle\left\langle\left(\lambda_{\mathrm{c}} \mu_{\mathrm{c}}\right) \kappa_{\mathrm{c}}^{\prime} L_{\mathrm{c}} ;(Q 0) L_{\mathrm{r}} \|(\lambda \mu) \kappa L\right\rangle .
\end{aligned}
$$

If the state of nucleus $A$ is approximated by the weak-coupling wave function, (2a) or (2b), the spectroscopic amplitude, $A_{\bar{L}_{\mathrm{r}}}^{Q}$, for the dissociation of nucleus $A$ into an $\alpha$-particle and a residual nucleus in the state specified by $\phi\left(\xi_{c}\right)$ is given by

$$
A_{\mathcal{Q}_{\mathrm{r}}}=1 / N_{\mathcal{Q}_{\mathrm{r}}}
$$

[See eq. (3.27) of ref. ${ }^{4}$ ).] Although extensions to more complicated relative motion wave functions are in principle straightforward, it should be emphasized that eqs. (6) and (7) give the $\alpha$-spectroscopic amplitude in harmonic oscillator approximation, since the relative motion function with quantum numbers $Q\left(=2 N_{\mathrm{r}}+L_{\mathrm{r}}\right)$ and $L_{\mathrm{r}}$ 
has been approximated by a harmonic oscillator function of definite SU(3) symmetry $(Q 0)$.

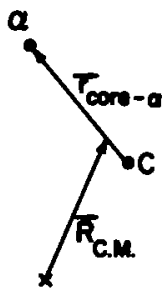

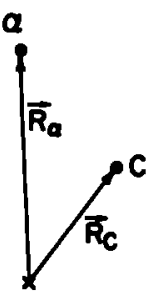

(a)
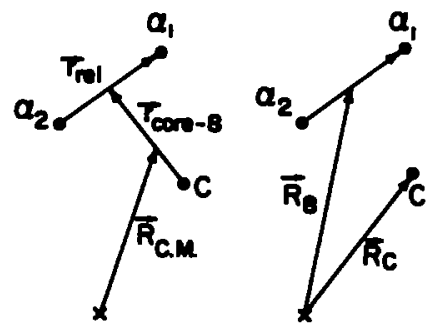

(b)

Fig. 1. Relative and cluster c.m. coordinates.

The method to be used to calculate elements of the operator $1-K$ makes use of a "cluster-like" wave function $\Psi^{\prime \prime}$ introduced by Ichimura et al. ${ }^{4}$ ) in which the relative motion function $\Phi^{(0)}\left(r_{c-a}^{\prime}\right)$ of eq. (1) is replaced by a function $\Phi^{\left(Q^{(0)}\right.}\left(R_{a}^{\prime}\right)$ of the coordinate $\boldsymbol{R}_{\alpha}^{\prime}=[4 \mathrm{m \omega} / \hbar]^{t} \boldsymbol{R}_{\alpha}$, and in which the internal cluster function is multiplied by a wave function of 0 s excitation for the c.m. motion of the core nucleus, $\Phi^{(00)}\left(R_{\mathrm{c}}\right)$; see fig. la.

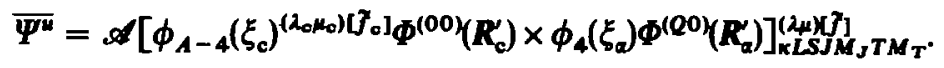

For these cluster-like (barred) functions overlaps are easy to calculate by projecting them onto totally antisymmetric shell-model states:

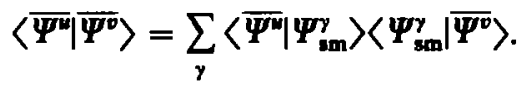

For states of a few $h \omega$ of oscillator excitation the sum over shell-model states $\gamma$ is restricted to a small number of terms since the overlaps are diagonal in the SU(3) quantum numbers $(\lambda \mu)$ and in the total number of oscillator quanta; and the shellmodel states are restricted to those with the same core configurations found in the cluster state. Note, however, that functions $\overline{\Psi^{\mu}}, \overline{\Psi^{v}}$ based on different $\left(\lambda_{\mathrm{c}} \mu_{\mathrm{c}}\right)$ are not orthogonal to each other.

The final step in the calculation of matrix elements of $1-K$ then makes use of a transformation from the cluster center of mass coordinates $\boldsymbol{R}_{\alpha}, \boldsymbol{R}_{\mathrm{c}}$, to relative coordinates $\boldsymbol{r}_{\mathrm{c}-\mathrm{x}}$ and $\boldsymbol{R}_{\mathrm{c}, \mathrm{m}}$, the coordinate of the c.m. of the whole system (fig. 1a), by which the cluster-like $\left|\Psi^{T}\right\rangle$ for states of oscillator excitation $k \hbar \omega$ are expanded in terms of true cluster states $\left|\Psi^{\eta}\right\rangle$ of oscillator excitation $k h \omega,(k-1) \hbar \omega, \ldots, 0 \hbar \omega$. This is a technique introduced by Ichimura $e t$ al. ${ }^{4}$ ) and also used by Aljadir ${ }^{50}$ ), which however is greatly simplified by the use of SU(3) strong-coupled wave functions and SU(3) recoupling techniques. [It is interesting to note that integral transform 
techniques for the calculation of resonating group norm kernels have made use of a similar intermediate step. Here also a transformation from cluster center of mass coordinates to relative cluster coordinates is accomplished in the last step of the calculation $\left.{ }^{5}\right)$.]

Both of the basic steps of the calculation, the transformation from $\left|\overline{\Psi^{n}}\right\rangle$ to $\left|\Psi^{\prime \prime}\right\rangle$, and the calculation of the overlaps $\left\langle\Psi_{\mathrm{SM}}^{\gamma}\right| \overline{\left.\Psi^{\prime}\right\rangle}$, make use of an expansion of harmonic oscillator wave functions $\Phi\left(\rho^{\prime}\right)$ in a variable $\rho^{\prime}$ in terms of harmonic oscillator functions $\Phi\left(r_{i}^{\prime}\right)$, or $\Phi\left(R_{k}^{\prime}\right)$, where the variables $\rho^{\prime}$ and $r_{i}^{\prime}$, or $R_{k}^{\prime}$, are related by unitary transformations,

where, e.g., for some index $j$,

$$
\boldsymbol{\rho}_{j}^{\prime}=\sum_{i} \boldsymbol{r}_{i}^{\prime} u_{i j}^{\prime}
$$

$$
\begin{aligned}
\rho^{\prime} & =\left[\frac{4(A-4)}{A} \frac{m \omega}{h}\right]^{\sharp} r_{c-\alpha} \equiv r_{c-\alpha}^{\prime} \\
& =\left[\frac{A-4}{4 A}\right]^{t}\left(r_{A-3}^{\prime}+r_{A-2}^{\prime}+r_{A-1}^{\prime}+r_{A}^{\prime}\right)-\left[\frac{4}{A(A-4)}\right]^{t}\left(r_{1}^{\prime}+r_{2}^{\prime}+\ldots+r_{A-4}^{\prime}\right) .
\end{aligned}
$$

Or, with another choice of $\rho^{\prime}$,

Alternately

$$
\begin{gathered}
\rho^{\prime}=[4 m \omega / \hbar]^{ \pm} R_{\alpha} \equiv R_{\alpha}^{\prime}, \\
R_{\alpha}^{\prime}=\sqrt{\frac{1}{4}\left(r_{A-3}^{\prime}+r_{A-2}^{\prime}+r_{A-1}^{\prime}+r_{A}^{\prime}\right) .}
\end{gathered}
$$

$$
\begin{aligned}
& \boldsymbol{R}_{a}^{\prime}=\left[\frac{A-4}{A}\right]^{\frac{1}{2}} \boldsymbol{r}_{\mathrm{c}-\alpha}^{\prime}+\left[\begin{array}{l}
4 \\
A
\end{array}\right]^{\frac{1}{2}} \boldsymbol{R}_{\mathrm{c} . \mathrm{s}}^{\prime} \\
& =\left[\frac{A-4}{A}\right]^{\frac{1}{2}}\left(\frac{4(A-4)}{A} \frac{m \omega}{h}\right)^{\frac{1}{t}} r_{\mathrm{c}-\alpha}+\left[\frac{4}{A}\right]^{\frac{1}{2}}\left(\frac{A m \omega}{h}\right)^{\frac{1}{t}} \mathrm{R}_{\mathrm{cm}} .
\end{aligned}
$$

It will be convenient to express single particle harmonic oscillator wave functions in terms of polynomials in harmonic creation operators, $\eta_{i}$, for the $i$ th particle, acting on the oscillator ground state $|0\rangle$ :

$$
\Phi_{n l m}\left(\boldsymbol{r}_{i}[m \omega / \hbar]^{\frac{1}{t}}\right)=P_{l m}^{(q 0)}\left(\boldsymbol{\eta}_{i}\right)|0\rangle,
$$

with similar expressions for oscillator functions in the variables $\rho$. In place of the principal quantum numbers $n, S U(3)$ representation labels $(q 0)$ will always be used, where the number of oscillator quanta $q=2 n+l$. The basic expansion of a harmonic oscillator function $\Phi\left(\rho_{j}^{\prime}\right)$ in terms of functions $\Phi\left(r_{i}^{\prime}\right)$ has its simplest form in an SU(3) coupled representation. For example,

$$
\begin{aligned}
\Phi_{L M}^{(Q O)}\left(\rho_{j}^{\prime}=\right. & \left.\sum_{i}^{A} r_{i}^{\prime} u_{i j}\right)=\sum_{\substack{q_{1} q_{2} \cdots \cdots q_{A} \\
\left(\Sigma_{2}=Q\right)}}\left[\frac{Q !}{\prod_{i=1}^{A}\left(q_{i} !\right.}\right]^{t} \prod_{i=1}^{A}\left(u_{i j}\right)^{q_{i}} \\
& \times\left[\ldots\left[\left[P^{\left(q_{1} 0\right)}\left(\eta_{1}\right) \times P^{\left(q_{2} 0\right)}\left(\eta_{2}\right)\right]^{\left(q_{1}+q_{2}, 0\right)} \times P^{\left(q_{3} 0\right)}\left(\eta_{3}\right)\right]^{\left(q_{1}+q_{2}+q_{3}, 0\right)} \times \ldots\right. \\
& \left.\left.\times P^{\left(q_{A} 0\right)}\left(\eta_{A}\right)\right]_{L M}^{(O)} \mid 0\right),
\end{aligned}
$$


a result given by Kramer $^{31}$ ) which makes use of the transformation properties of the $A$-particle harmonic oscillator function under the direct product group $\mathrm{U}(A) \times \mathrm{U}(3)$, where Kramer's result has been transcribed here into $\mathrm{SU}(3)$ coupled language. The square brackets [ ] indicate SU(3) coupling. For example,

$$
\begin{aligned}
& \left.\left[P^{\left(q_{1} 0\right)}\left(\eta_{1}\right) \times P^{\left(q_{2} 0\right)}\left(\eta_{2}\right)\right]\right]_{i_{m}}^{\left(q_{1}+q_{2}, 0\right)} \\
& \quad=\sum_{l_{1} l_{2}}\left\langle\left(q_{1} 0\right) l_{1} ;\left(q_{2} 0\right) l_{2} \|\left(q_{1}+q_{2}, 0\right) l\right\rangle \sum_{m_{1} m_{2}}\left\langle l_{1} m_{1} l_{2} m_{2} \mid l m\right\rangle P_{l_{1} m_{1}}^{\left(q_{1} 0\right)}\left(\eta_{1}\right) P_{l_{2} m_{2}}^{\left(q_{2} 0\right)}\left(\eta_{2}\right) .
\end{aligned}
$$

Since all SU(3) couplings are "stretched" couplings of commuting operators, the order of the couplings in eq. (16) can be rearranged at will (without change of phase). Note also that much of the simplicity of eq. (16) disappears in the analogous expression in an angular momentum coupled representation.

Similar expansions making use of the unitary transformations of eqs. (12) and (13) in four and two dimensions, respectively, can be used to expand harmonic oscillator functions $\Phi\left(R_{a}^{\prime}\right)$ in terms of harmonic oscillator functions in the variables $r_{A-3}^{\prime}$, $r_{A-2}^{\prime}, r_{A-1}^{\prime}$ and $r_{A}^{\prime}$, or the variables $r_{c-\alpha}^{\prime}$ and $R_{c . m .}^{\prime}$. For example, the transformation

$$
\begin{aligned}
\Phi_{L M}^{(Q 0)}\left(R_{\alpha}^{\prime}\right)=\sum_{\substack{Q_{1} Q_{2} \\
\left(Q_{1}+Q_{2}=Q\right)}}\left[\frac{Q !}{Q_{1} ! Q_{2} !}\right]^{ \pm}\left[\frac{A-4}{A}\right]^{+Q_{1}} & {\left[\frac{4}{A}\right]^{+Q_{2}} } \\
& \times\left[\Phi^{\left(Q_{1} 0\right)}\left(r_{c-\alpha}^{\prime}\right) \times \Phi^{\left(Q_{2} 0\right)}\left(R_{c . m .}^{\prime}\right)\right]_{L M}^{(Q 0)},
\end{aligned}
$$

together with the trivial transformation

$$
\Phi^{(00)}\left(R_{\mathrm{c}}^{\prime}\right) \equiv \Phi^{(00)}\left(r_{\mathrm{c}-\mathrm{q}}^{\prime}\right) \times \Phi^{(00)}\left(R_{\mathrm{c} . \mathrm{m} .}^{\prime}\right)
$$

can be used to express the cluster-like function $\overline{\varphi^{u}}$ in terms of translationally invariant true cluster functions $\Psi^{*}$ in SU(3) strong-coupled form:

$$
\begin{aligned}
& \overline{\Psi^{u}}=\sum_{\substack{Q_{2}=0 \\
\left(Q_{1}+Q_{2}=Q\right)}}\left[\frac{Q !}{Q_{1} ! Q_{2} !}\right]^{\frac{1}{2}}\left[\frac{A-4}{A}\right]^{\frac{1}{Q_{1}}}\left[\frac{4}{A}\right]^{\frac{1}{2} Q_{2}}
\end{aligned}
$$

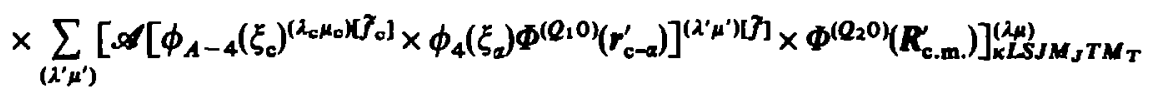

$$
\begin{aligned}
& \times U\left(\left(\lambda_{c} \mu_{c}\right)\left(Q_{1} 0\right)(\lambda \mu)\left(Q_{2} 0\right) ;\left(\lambda^{\prime} \mu^{\prime}\right)(Q 0)\right),
\end{aligned}
$$

where the $U$-coefficient is an SU(3) Racah coefficient in unitary form. [The notation is a generalization of that for angular momentum coupling, see refs. $\left.{ }^{31},{ }^{34}\right)$.] These SU(3) recoupling coefficients involving the simple stretched coupling of $\left(Q_{1} 0\right) \times\left(Q_{2} 0\right)$ have a particularly simple form (see the appendix). The SU(3) recoupling transformation, illustrated in fig. 2 , is needed to expand the right hand side of (19) in terms of true cluster functions coupled to SU(3) representations $\left(\lambda^{\prime} \mu^{\prime}\right)$. The oscillator functions $\Phi\left(R_{c . m}^{\prime}.\right)$ carrying the excitations of the c.m. motion 


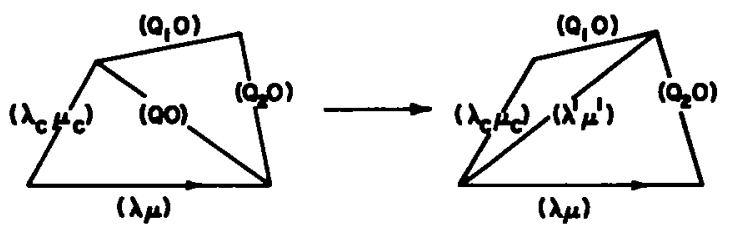

Fig. 2. The SU(3) recoupling transformation for eq. (19). The triangles represent SU(3) coupling.

are totally symmetric so that $\mathscr{A c t s}$ only on the true cluster functions. The c.m. excitations range from $Q_{2}=0$ (the nonspurious component of $\overline{\Psi^{2}}$ ) to $Q_{2}=k$, where $k$ is the total number of harmonic oscillator excitations in the cluster-like function. Since c.m. excitation functions with different values of $Q_{2}$ are orthogonal to each other and since the overlaps of both cluster-like and true cluster functions are diagonal in the SU(3) quantum numbers, $(\lambda \mu)$, the overlaps $\left\langle\overline{\Psi^{x}} \mid \overline{\Psi^{\nu}}\right\rangle$ are simply related to the corresponding $\left\langle\Psi^{u} \mid \Psi^{\nu}\right\rangle$ :

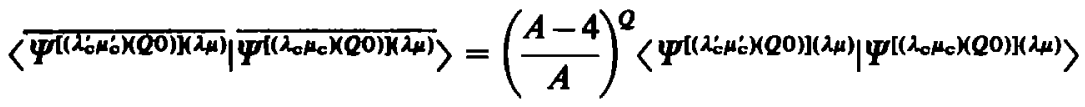

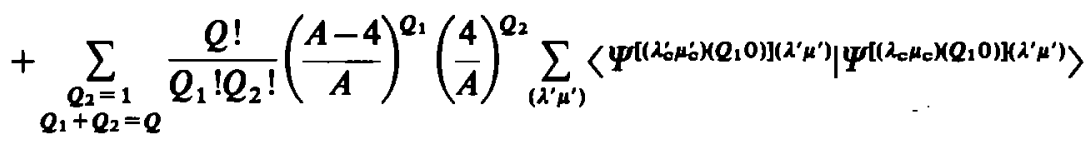

$$
\begin{aligned}
& \times U\left(\left(\lambda_{\mathrm{c}}^{\prime} \mu_{\mathrm{c}}^{\prime}\right)\left(Q_{1} 0\right)(\lambda \mu)\left(Q_{2} 0\right) ;\left(\lambda^{\prime} \mu^{\prime}\right)(Q 0)\right) U\left(\left(\lambda_{\mathrm{c}} \mu_{\mathrm{c}}\right)\left(Q_{1} 0\right)(\lambda \mu)\left(Q_{2} 0\right) ;\left(\lambda^{\prime} \mu^{\prime}\right)(Q 0)\right) .
\end{aligned}
$$

[It has been assumed for simplicity that the core states $\left(\lambda_{\mathrm{c}}^{\prime} \mu_{\mathrm{c}}^{\prime}\right)$ and $\left(\lambda_{\mathrm{c}} \mu_{\mathrm{c}}\right)$ carry the same number of oscillator quanta.]

In the $\mathrm{SU}(3)$ strong-coupled single channel approximation [based on a single core state $\left(\lambda_{c} \mu_{c}\right)$ ], the above relates the normalization coefficients of the clusterlike states, $\bar{N}_{(\lambda \mu)}^{Q}$, to the normalization coefficients of the true cluster states, $N_{(\lambda \mu)}^{Q}$ (the normalized functions are $N \Psi^{u}$ and $N \Psi^{u}$, respectively). In this case eq. (20) becomes

$$
\begin{aligned}
& \frac{1}{\left[\bar{N}_{(\lambda \mu)}^{Q}\right]^{2}}=\frac{1}{\left[N_{(\lambda \mu)}^{Q}\right]^{2}}\left(\frac{A-4}{A}\right)^{Q} \\
& +\sum_{\substack{Q_{2}=1 \\
Q_{1}+Q_{2}=Q}} \frac{Q !}{Q_{1} ! Q_{2} !}\left(\frac{A-4}{A}\right)^{Q_{1}}\left(\frac{4}{A}\right)^{Q_{2}} \sum_{\left(\lambda^{\prime} \mu^{\prime}\right)} \frac{1}{\left[N_{\left(\lambda^{\prime} \mu^{\prime}\right)}^{Q^{\prime}}\right]^{2}} U^{2}\left(\left(\lambda_{c} \mu_{c}\right)\left(Q_{1} 0\right)(\lambda \mu)\left(Q_{2} 0\right) ;\left(\lambda^{\prime} \mu^{\prime}\right)(Q 0)\right) .
\end{aligned}
$$

Hence $N_{(\lambda \mu)}^{Q}$ is determined from the norm of the cluster-like state if the normalization constants for states $Q_{1}<Q$ are known. If the core state is that of an s-d shell nucleus in its ground-state configuration, e.g., the cluster function with $Q=8+k$ corresponds to a shell-model function of $k$-units of oscillator excitation. Its norm 
is thus known if norms for cluster states corresponding to oscillator excitations $(k-1) \hbar \omega, \cdots, 0 h \omega$ have previously been calculated. Since any application will usually require knowledge of norm and overlap kernels up to a certain value of $k$, the chain nature of this calculational technique will not be a disadvantage.

\section{Alpha clusters and core excitations in s-d shell nuclei}

Since there is considerable experimental information for $\alpha$-transfer reactions to negative parity and other core-excited states in s-d shell nuclei, states of a few hw excitation in these nuclei are of special interest, particularly since deformed, low-lying core-excited states may have large overlaps with $\alpha$-cluster states based on cores of good SU(3) and supermultiplet symmetry. In order to be able to make predictions for both the weak and strong SU(3) coupling limits or specific intermediate coupling wave functions, overlap and norm factors are needed for all possible $(\lambda \mu)$ in the coupling of $\left(\lambda_{\mathrm{c}} \mu_{\mathrm{c}}\right) \times(Q 0)$.

\subsection{AN EXAMPLE: STATES OF 1 ho EXCITATION IN ${ }^{21} \mathrm{Ne}$}

Alpha cluster wave functions for ${ }^{21} \mathrm{Ne}$ are particularly simple since the ${ }^{17} \mathrm{O}$ core in its ground-state configuration is limited to the single SU(3) representation (20). The calculations for ${ }^{21} \mathrm{Ne}$ nevertheless fully illustrate the method of calculation. The cluster-like state is now

$$
\Phi^{*}=\mathscr{A}\left[\phi_{17}\left(\xi\left({ }^{17} \mathrm{O}\right)\right)^{(20)[1]} \Phi^{(00)}\left(R_{17}^{\prime}\right) \times \phi_{4}\left(\xi_{\alpha}\right) \Phi^{(00)}\left(R_{\alpha}^{\prime}\right)\right]_{\ldots}^{(2 \mu)(1]} .
$$

States with $Q<8$ are Pauli-forbidden. The normalization factors for states with $Q=8$ (i.e. states in the ground-state configuration of ${ }^{21} \mathrm{Ne}: \mathrm{s}^{4} \mathrm{p}^{12} \mathrm{sd}^{5}$ ) follow from refs. ${ }^{4,34}$ ), and in the notation of these references are

$$
\frac{1}{N_{(\lambda \mu)}^{8}}=\left(\frac{21}{17}\right)^{4} G\left(\mathrm{sd}^{4}\right)\left\langle(\lambda \mu)\|\| \chi_{(80)}^{+}\|\|(20)\right\rangle,
$$

with $(\lambda \mu)=(81),(62)$. The state with $(\lambda \mu)=(10,0)$ is Pauli-forbidden. For states of $1 \hbar \omega$ excitation $(Q=9)$ the possible $(\lambda \mu)$ of $(11,0),(91),(72)$ are all allowed. The states $\Psi^{u}$ will project onto shell-model states

$$
\Psi_{\mathrm{Sm}}^{y}=\Psi\left(\mathrm{s}^{4} \mathrm{p}^{12}(\mathrm{sd})^{4}\left(\lambda_{4} \mu_{4}\right)\left[f_{4}\right] \times(\mathrm{pf})^{1}(30)[1]\right)_{\ldots}^{(\lambda \mu)[1]},
$$

with $\left[f_{4}\right]=[0]$ (space symmetry $\left.[4]\right),(\lambda \mu)=(80),(42) \ldots$, and with $\left[f_{4}\right]=[211]$ (space symmetry [31]), $(\lambda \mu)=(61),(42) \ldots .$. The sum over $\gamma$ in the projection onto shell-model states (eq. (9)) thus involves a small number of terms.

$$
\begin{array}{llll}
\text { For }(\lambda \mu)=(11,0), & \gamma: & {\left[f_{4}\right]\left(\lambda_{4} \mu_{4}\right)=[0](80) .} \\
\text { For }(\lambda \mu)=(91), & \gamma: & {\left[\tilde{f}_{4}\right]\left(\lambda_{4} \mu_{4}\right)=[0](80),[211](61) .} \\
\text { For }(\lambda \mu)=(72), & \gamma: & {\left[f_{4}\right]\left(\lambda_{4} \mu_{4}\right)=[0](80) \text { and }(42),[211](61) \text { and }(42) .}
\end{array}
$$


It will be convenient to express the ${ }^{17} \mathrm{O}$ wave function in $\Psi^{4}$ as

$$
\mathscr{A}^{\prime}\left[\Psi\left(r_{1}^{\prime}, \ldots, r_{16}^{\prime}\right)^{(00)[0]} \Psi\left(r_{17}^{\prime}\right)^{(20)(1)}\right],
$$

and let the antisymmetrizers $\mathscr{A}$ and $\mathscr{A}^{\prime}$ act to the left in the overlap $\left\langle\Psi_{\mathrm{sun}} \mid \Psi^{m}\right\rangle$. In the expansion of $\Phi^{(90)}\left(R_{x}^{\prime}=\frac{1}{2}\left(r_{18}^{\prime}+r_{19}^{\prime}+r_{20}^{\prime}+r_{21}^{\prime}\right)\right)$ according to eq. (15) only four terms can contribute to the overlap with the shell-model states, the terms with $q_{1} q_{2} q_{3} q_{4}=2223,2232,2322,3222$. From the total symmetry of $\Phi\left(R_{a}\right)$, and the antisymmetry of $\phi\left(\xi_{a}\right)$ and $\Psi_{\text {sau }}$, it follows that these four terms contribute to the overlap with the same sign, so that

$$
\begin{aligned}
& \left\langle\psi_{\mathrm{SM}}^{\gamma} \mid \propto\left[\phi_{17}\left(\xi^{17} \mathrm{O}\right)^{\left(20 \times 11^{1}\right.} \Phi^{(00)}\left(\boldsymbol{R}_{17}^{\prime}\right) \times \phi_{4}\left(\xi_{\alpha}\right) \Phi^{(00)}\left(\boldsymbol{R}_{\alpha}^{\prime}\right)\right]^{(\lambda \mu)[1]}\right\rangle \\
& =\left[\left(\begin{array}{l}
21 \\
17
\end{array}\right)\left(\begin{array}{l}
17 \\
16
\end{array}\right)\right]^{ \pm}\left[\frac{9 !}{2 ! 2 ! 2 ! 3 !}\right]^{\sharp}\left(\frac{1}{2}\right)^{9} 4\left\langle\Psi_{\mathrm{SM}}^{\gamma}\right| \Psi\left(\mathrm{s}^{4} \mathrm{p}^{12}, r_{1}^{\prime} \ldots r_{16}^{\prime}\right)^{(00) 0]} \\
& \left.\left.\left.\times \Psi\left(\mathrm{sd}^{1}, r_{17}^{\prime}\right)^{(20)[1]}\left[\Psi(\mathrm{sd})^{3}, r_{18}^{\prime} \ldots r_{20}^{\prime}\right)^{(60)\left(1^{3}\right]} \times \Psi\left((\mathrm{pf})^{1}, r_{21}^{\prime}\right)^{(30)(11}\right]^{(90)(00}\right]_{\ldots}^{(2 \mu)(1]}\right\rangle,
\end{aligned}
$$

where the binomial coefficients come from the action of $\mathscr{A}, \mathscr{A}^{\prime}$, and the last factor of four follows from the combination of terms for the four possible $q_{1} q_{2} q_{3} q_{4}$. To take the overlap it will now be convenient to decompose the shell-model wave function according to configurations

$$
\begin{aligned}
& \Psi_{\mathrm{SM}}=\mathscr{A}^{\prime \prime} \Psi\left(\mathrm{s}^{4} \mathrm{p}^{12}, r_{1}^{\prime} \ldots r_{16}^{\prime}\right)^{(00)(0)}\left[\Psi((\mathrm{sd}))^{4} r_{17}^{\prime} \ldots r_{20}^{\prime}\right)^{\left.\left(\lambda_{A} \mu_{4}\right) f_{4}\right]} \\
&\left.\left.\times \Psi(\mathrm{pf})^{1} r_{21}^{\prime}\right)^{(30)(1)}\right]^{(4))[1]},
\end{aligned}
$$

where the antisymmetrizer $\mathscr{A}^{\prime \prime}$ antisymmetrizes between the three groups of particles, $\left(\mathrm{s}^{4} \mathrm{p}^{12}\right)$, (sd) and $(\mathrm{pf})^{1}$. However, only the identity operator within $\mathscr{A}^{\prime \prime}$ can contribute to the overlap with the right hand ket of eq. (24). The normalization coefficient of $\mathscr{A}^{\prime \prime}$ contributes a factor $[21 ! / 16 ! 4 ! 1 !]^{-\frac{1}{3}}$ to the overlap. Finally, the SU(3)-SU(4) recoupling transformation illustrated by fig. 3 is needed to relate the overlap of eq. (24) to a simple sd shell c.f.p.:

$$
\begin{aligned}
& \left.\left\langle\Psi\left(r_{17}^{\prime} \ldots r_{20}^{\prime}\right)^{\left(\lambda_{4} \mu_{4}\right)\left[f_{4}\right]}\left[\Psi\left(r_{17}^{\prime}\right)^{(20)[1]} \times \Psi^{\prime} r_{18}^{\prime} \ldots r_{20}^{\prime}\right)^{(60)\left(1^{3}\right]}\right]^{\left(\lambda_{4} \mu_{4}\right)\left[f_{4}\right]}\right\rangle \\
& =\frac{1}{\left[\left(\begin{array}{l}
4 \\
3
\end{array}\right)\right]^{\frac{3}{4}}}\left\langle\left(\lambda_{4} \mu_{4}\right)\left[f_{4}\right]\|\| \chi^{+}(60)\|\|(20)[1]\right\rangle,
\end{aligned}
$$

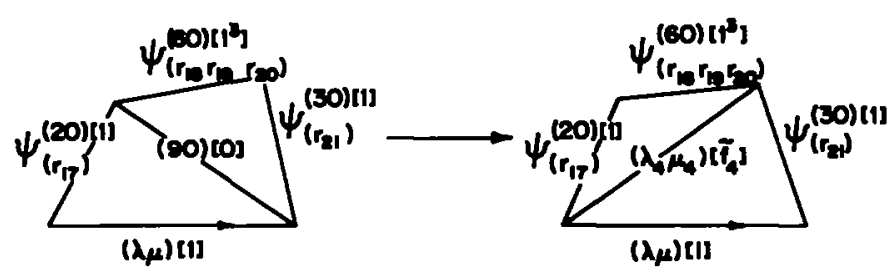

Fig. 3. The SU(3), SU(4) recoupling transformation needed for the overlap (24). The triangles represent both SU(3) and SU(4) coupling. 
which is here expressed in terms of the quadruple-barred matrix elements tabulated in ref. ${ }^{34}$ ). Thus

$$
\begin{aligned}
& \left\langle\Psi_{\mathrm{SM}}^{\gamma} \mid \Psi^{u}\right\rangle=\left\{\left[\frac{\left(\begin{array}{l}
21 \\
17
\end{array}\right)\left(\begin{array}{l}
(17 \\
(16
\end{array}\right)}{(16)\left(\begin{array}{l}
3 \\
4
\end{array}\right)}\right]^{\frac{1}{t}}\left[\frac{9 !}{2 ! 2 ! 2 ! 3 !}\right]^{\ddagger} \frac{4}{2^{9}} \frac{1}{\left[\left(_{3}^{4}\right)\right]^{\frac{1}{2}}}\right\}\left\langle\left(\lambda_{4} \mu_{4}\right)\left[f_{4}\right]\|\| \chi^{+}(60)\|\|(20)[1]\right\rangle \\
& \times U\left((20)(60)(\lambda \mu)(30) ;\left(\lambda_{4} \mu_{4}\right)(90)\right) U\left([1]\left[1^{3}\right][1][1] ;\left[\tilde{f}_{4}\right][0]\right) \text {. }
\end{aligned}
$$

The factor in curly brackets is $[(9 ! / 2 ! 2 ! 2 ! 3 !) 4]^{ \pm} / 2^{9}$, the $G$-factor of Ichimura et al. $\left.{ }^{4}\right)$, $G\left((\mathrm{sd})^{3}(\mathrm{pf})^{1}\right)$. The first $U$-coefficient is an SU(3) Racah coefficient, which in more general cases is readily available through the code of Akiyama and Draayer ${ }^{32}$ ). Note that for most calculations with single $\alpha$-clusters, such coefficients generally involve a simple stretched coupling such as $(60) \times(30) \rightarrow(90)$. For such coefficients a simple algebraic expression is available (appendix). In this particular example, in addition, all representations of $U(3)$ are two-rowed so that this particular SU(3) $U$-coefficient can be read from tables of SU(2) Racah coefficients. The last $U$ coefficient is an SU(4) Racah coefficient of a particularly simple form thanks to the $\mathrm{SU}(4)$-irreducible character [0] of the $\alpha$-particle. Its magnitude is given by simple SU(4) dimension ratios ${ }^{33}$ ). A consistent set of phases is obtained if all unitary group recoupling coefficients are interpreted as permutation group recoupling coefficients ${ }^{52,33}$ ), and if the permutation group phase conventions of Kramer ${ }^{33}$ ) are adopted. The ordinary SU(2) phase conventions and the SU(3) phase conventions of Draayer and Akiyama ${ }^{31}$ ) are consistent with these. However, the SU(4) phase conventions of refs. ${ }^{33,34}$ ) must be modified. In the Kramer phase convention, a multinucleon p-shell c.f.p. for the SU(3)-SU(4) coupled functions is given by simple permutation group $\left(S_{n}\right)$ dimension ratios:

$$
\begin{aligned}
& \left\langle\left[\Psi\left(p^{n_{1}}, r_{1}^{\prime} \ldots r_{n_{1}}^{\prime}\right)^{\left(\lambda_{1} \mu_{1}\right)\left[\gamma_{1}\right]} \times \Psi\left(p^{n_{2}}, r_{n_{1}+1}^{\prime} \ldots r_{n}^{\prime}\right)^{\left(\lambda_{2} \mu_{2}\right)\left[\gamma_{2} 2\right.}\right]_{\ldots}^{\left(\lambda_{\mu} \mu\right)\left[\gamma^{2}\right)}\right. \\
& \times\left|\Psi\left(p^{n}, r_{1} \ldots r_{n=n_{1}+n_{2}}^{\prime}\right)^{(\lambda \mu)\left[{ }^{\gamma}\right]}\right\rangle \\
& =(-1)^{\phi}\left[\frac{\operatorname{dim}_{S_{n_{1}}}\left[f_{1}\right] \operatorname{dim}_{S_{n_{2}}}\left[f_{2}\right]}{\operatorname{dim}_{S_{n}}[f]}\right]^{t} ; \quad \text { with }(-1)^{\phi}=\prod_{1=s<t=n_{1}+1}^{n} \varepsilon_{s t}
\end{aligned}
$$

where [f] describes the full three-rowed Young tableaux which give the space symmetry of the p-shell wave functions, from which the SU(3) and SU(4) quantum numbers can be read. The phase factor $\phi$ follows from the signs $\left(\varepsilon_{z t}\right)$ of the axial distances of the particles numbered $s, t$. [See eqs. (3.2)-(3.4) of ref. ${ }^{53}$ ).] Here $s$ ranges from 1 to $n-1$ with $s<t$, while $t$ ranges from $n_{1}+1$ to $n$. The particle indices $n_{1}+1$ to $n$, whose removal from $[f]$ leaves the tableau $\left[f_{1}\right]$, must be placed in the tableau for $[f]$ such that a shift up and/or to the left through an emptied tableau for $\left[f_{1}\right]$ of the labels $n_{1}+1, n_{1}+2, \ldots$, in order, leads to a tableau of shape $\left[f_{2}\right]$. The analogous phase and dimension factors for the s-d shell multinucleon c.f.p. are buried within the definition of the quadruple-barred reduced matrix elements of 
ref. ${ }^{34}$ ), eqs. (23) and (25). It is noteworthy that the norm factors for single $\alpha$-clusters in the single channel approximation [based on a single core state $\left(\lambda_{\mathrm{c}} \mu_{\mathrm{c}}\right)$ ] are completely free of phase difficulties, since all steps of the calculation involve only the squares of overlaps, see eqs. (9) and (21). In more complicated cases the requirement that the eigenvalues of $1-K$ must be zero for redundant or spurious states often serves as a valuable check on phases.

The norm factors $N$ for the cluster-like states in ${ }^{21} \mathrm{Ne}$ with $Q=9$ follow from eqs. (26) and (9). The norm factors $N$ for the true cluster states follow from eq. (21). The state with $(\lambda \mu)=(11,0)$ has no spurious c.m. component. Terms with $Q_{2}=1$ in eq. (21) are missing, and $\left[1 / N_{(11,0)}^{9}\right]^{2}=\left(\frac{21}{17}\right)^{9}\left[1 / N_{(11,0)}^{9}\right]^{2}$. For the state with $(\lambda \mu)=(91)$, a term with $Q_{2}=1$ and $\left(\lambda^{\prime} \mu^{\prime}\right)=(81)$ makes a contribution to $N_{(91)}^{9}$, since the cluster-like state (91) contains a spurious component with 1 h $\omega$ of c.m. excitation based on the $0 \hbar \omega$ state with $\left(\lambda^{\prime} \mu^{\prime}\right)=(81)$. Similarly, for the state with $(\lambda \mu)=(72)$ a term with $Q_{2}=1$ and $\left(\lambda^{\prime} \mu^{\prime}\right)=(62)$ in eq. (21) makes a contribution to $N_{(72)}^{9}$.

\subsection{APPLICATIONS}

The norm factors needed for states of $1 \hbar \omega$ excitation $(Q=9)$ in light s-d shell nuclei are shown in table 1 . In most of these nuclei a core state built from a single $\left(\lambda_{\mathrm{c}} \mu_{\mathrm{c}}\right)$ is a good approximation. The one exception may be the core ${ }^{18} \mathrm{O}$ since both $\left(\lambda_{c} \mu_{c}\right)=(40)$ and (02) are significant components in the [44442] space symmetry piece of the ${ }^{18} \mathrm{O}$ ground state. Norm and overlap factors for both representations are therefore shown in table 1 . Of the SU(3) strong-coupled cluster states in ${ }^{22} \mathrm{Ne}$, however, only the state with $(\lambda \mu)=(82)$ for $Q=8$ and (92) for $Q=9$ can be built from a linear combination of $\left(\lambda_{c} \mu_{c}\right)=(40)$ and (02) core states. For $Q=8$ there is only a single Pauli-allowed state with $(\lambda \mu)=(82)$. The operator $1-K$ has one eigenvalue of zero. The eigenvector associated with the other nonzero, eigenvalue gives the single (normalized) nonspurious state

$$
\left(\frac{18}{22}\right)^{4} \frac{2^{7}}{69 \sqrt{15}}\left\{\sqrt{55} \Psi^{[(40) \times(80) \mathrm{K} 82)}-\sqrt{14} \Psi^{[(02) \times(80)](82)}\right\},
$$

where $\Psi\left[\left(\lambda_{0} \mu_{0}\right) \times(Q)\right](\lambda \mu)$ is shorthand notation for the cluster state of eq. (1). The single allowed state can be described in this "coupled channel" form or, equally well, as the simple (normalized) cluster state

$$
\left(\frac{18}{22}\right)^{4} \frac{2^{7}}{5 \sqrt{33}} \Psi^{[(40) \times(80)\} 82)}
$$

The $\alpha$-spectroscopic amplitude for $\left({ }^{18} \mathrm{O}\right)_{0^{+}}+\alpha \rightarrow\left({ }^{22} \mathrm{Ne}\right)_{(82)^{+}}$is of course inde- 
TABLE 1

The $\alpha$-cluster norm factors in light s-d shell nuclei

\begin{tabular}{|c|c|c|c|c|c|}
\hline & $\left(\lambda_{a} \mu_{a}\right)$ & $\boldsymbol{Q}$ & $(\lambda \mu)$ & $\left\langle\Psi^{\prime \prime} \mid \Psi^{m}\right\rangle /(A \mid A-4)^{Q}$ & {$\left[1 / N_{[2 \mu]}^{e}\right]^{2}$} \\
\hline$A=21$ & (20) & $\begin{array}{l}8 \\
9\end{array}$ & $\begin{array}{l}(81) \\
(62) \\
(11,0) \\
(91) \\
(72)\end{array}$ & $\begin{array}{l}3^{2} \times 5^{2} \times 7 / 2^{15} \\
3^{5} \times 5 / 2^{15} \\
3^{3} \times 5 \times 7 / 2^{15} \\
3 \times 5 \times 59 / 2^{14} \\
3 \times 5 \times 7 \times 61 / 2^{17}\end{array}$ & $\begin{array}{l}0.26061 \\
0.20104 \\
0.19316 \\
0.36179 \\
0.32730\end{array}$ \\
\hline$A=22:$ & (40) & 8 & $\begin{array}{l}(82) \\
(63) \\
(44)_{1} \\
(44)_{2} \\
(82) \\
(71) \\
(60)_{1} \\
(60)_{2} \\
(11,1) \\
(92) \\
(73) \\
(54) \\
(92) \\
(81) \\
(70)\end{array}$ & $\begin{array}{l}\left.3 \times 5^{2} \times 11 / 2^{14 a}\right) \\
3^{3} \times 5^{2} / 2^{14} \\
0 \\
3^{3} \times 11 / 2^{13} \\
3 \times 5 \times 7 / 2^{13} a \\
3^{2} \times 5^{2} \times 7 / 2^{15} \\
0 \\
3^{2} \times 5 \times 13 / 2^{14} \\
3 \times 5 \times 7 \times 13 / 2^{13} \\
\left.3^{2} \times 5 \times 7 \times 11 / 2^{16 b}\right) \\
3^{2} \times 5 \times 163 / 2^{17} \\
3 \times 5^{2} \times 491 / 11 \times 2^{16} \\
\left.3^{3} \times 5 \times 7^{2} / 11 \times 2^{14 b}\right) \\
3^{3} \times 5 \times 7 / 2^{14} \\
3^{4} \times 5 \times 7 \times 13 / 2^{16} \times 11\end{array}$ & $\begin{array}{l}0.25075 \\
0.20516 \\
0 \\
0.18054 \\
0.06383 \\
0.23935 \\
0 \\
0.17780 \\
0.25353 \\
0.32179 \\
0.34060 \\
0.31090 \\
0.22339 \\
0.35105 \\
0.31115\end{array}$ \\
\hline$A=23$ & $(60)$ & 8 & $\begin{array}{l}(83) \\
(64) \\
(45) \\
(26) \\
(11,2) \\
(93) \\
(74) \\
(55) \\
(36)\end{array}$ & $\begin{array}{l}3^{2} \times 5 \times 11 / 2^{13} \\
3^{3} \times 11 / 2^{13} \\
3^{3} \times 5^{2} / 2^{14} \\
3^{4} \times 7 / 2^{14} \\
3 \times 7^{2} \times 13 / 2^{15} \\
3^{2} \times 7 \times 13 \times 89 / 2^{16} \times 23 \\
3^{2} \times 11 \times 949 / 2^{16} \times 23 \\
3 \times 5^{2} \times 11 \times 101 / 2^{16} \times 23 \\
3^{3} \times 5^{2} \times 7 \times 17 / 2^{16} \times 23\end{array}$ & $\begin{array}{l}0.27862 \\
0.16717 \\
0.18997 \\
0.15957 \\
0.32552 \\
0.26992 \\
0.34791 \\
0.30856 \\
0.29745\end{array}$ \\
\hline
\end{tabular}

) Overlap between $\left(\lambda_{0} \mu_{\mathrm{o}}\right)=(40)$ and $(02):-3 \times 5[770]^{1 / 2} / 2^{14}$.

b) Overlap between $\left(\lambda_{c} \mu_{c}\right)=(40)$ and $(02):-3^{4} \times 5 \times 7 / 2^{15}[33]^{1 / 2}$.

pendent of the description of this single (82) cluster state, and from either eqs. (28) or (29) leads to

$$
\begin{aligned}
A_{L=J}\left({ }^{18} \mathrm{O}+\alpha \rightarrow{ }^{22} \mathrm{Ne}\right)=\left(\frac{22}{18}\right)^{4} \frac{\sqrt{15}}{2^{7}}\left\{a_{(40)} \sqrt{55}\langle(40) 0 ;(80) L \|(82) \kappa J\rangle\right. \\
\\
\left.-a_{(02)} \sqrt{14}\langle(02) 0 ;(80) L \|(82) \kappa J\rangle\right\},
\end{aligned}
$$

where $a_{(\lambda \mu)}$ are the amplitudes of the different components in the $0^{+}$ground state of ${ }^{18} \mathrm{O}$. Eq. (30) is the ordinary shell-model prediction for the $\alpha$-spectroscopic amplitude [ref. $\left.\left.{ }^{35}\right)\right]$ for this state of $0 \hbar \omega$ oscillator excitation. Both states (29) and (28) have unit overlap with the single shell-model state $\Psi\left(\mathrm{s}^{4} \mathrm{p}^{12} \mathrm{sd}^{4}\right)$ with $(\lambda \mu)=(82)$. For 
$Q \geqq 9$ and symmetries $(\lambda \mu)$ for which there exist states of spurious c.m. excitation, the antisymmetrized cluster states pick out definite linear combinations of shell-model states ${ }^{54}$ ), free of c.m. excitation. The single and coupled-channel cluster descriptions will now in general lead to different final states. In ${ }^{22} \mathrm{Ne}$, e.g., with $Q=9,(\lambda \mu)=(92)$ the overlap operator $1-K$ has two nonzero eigenvalues in a coupled-channel representation based on core states with $\left(\lambda_{c} \mu_{c}\right)=(40)$ and $(02)$.

Since realistic $\alpha$-residual nucleus relative motion wave functions may require harmonic oscillator components including relatively high values of $Q$, norm factors up to $Q=12$ are shown, as an example, for ${ }^{24} \mathrm{Mg}$ based on a ${ }^{20} \mathrm{Ne}$ core of pure (80) symmetry (table 2). It is to be noted that the norm factors $N_{(\lambda \mu)}^{Q}$ for given $Q$ have very similar magnitudes, a result important for the approximations made in the orthogonality condition model ${ }^{19}$ ). There are, however, significant variations in $N_{(\lambda \mu)}^{Q}$ for states near the highest possible $\lambda$, which may be the most important components in the wave function. Note also that $N_{(\lambda \mu)}^{Q}$ tend toward unity as $Q$ increases. For very large $Q$ the separation between core and $\alpha$-clusters becomes so great that the effects of the antisymmetrizer become negligible.

Alpha spectroscopic amplitudes follow at once from the norm factors of tables 1 and 2. The work of Draayer et al. ${ }^{35,36-39}$ ) shows that predictions for $\alpha$-spectroscopic amplitudes based on the SU(3) strong coupling model [leading $(\lambda \mu)$ ] are in remarkably good agreement with experiment for states of $0 \hbar \omega$ excitation in light s-d shell nuclei. For core excited states, with $Q \geqq 9$, the weak coupling model may be a better approximation ${ }^{20,41-42}$ ). It is thus of interst to compare the predictions of the two

TABLE 2

Norm factors for ${ }^{24} \mathrm{Mg}$ based on a ${ }^{20} \mathrm{Ne}$ core with $\left(\lambda_{\mathrm{o}} \mu_{\mathrm{c}}\right)=(80)$

\begin{tabular}{clllll}
\hline$Q$ & $(\lambda \mu)$ & {$\left[1 / N_{(2 \mu)}^{Q}\right]^{2}$} & $Q$ & $(\lambda \mu)$ & {$\left[1 / N_{(2, \mu)}^{Q}\right]^{2}$} \\
\hline 8 & $(84)$ & 0.38601 & 11 & $(17,1)$ & 0.28273 \\
& $(46)$ & 0.33405 & & $(15,2)$ & 0.27550 \\
& $(08)$ & 0.29761 & & $(13,3)$ & 0.57580 \\
9 & & & $(11,4)$ & 0.50849 \\
& $(11,3)$ & 0.46058 & & $(95)$ & 0.60965 \\
& $(94)$ & 0.12282 & & $(76)$ & 0.57101 \\
& $(75)$ & 0.45839 & & $(57)$ & 0.57396 \\
& $(56)$ & 0.21156 & & $(38)$ & 0.55475 \\
& $(37)$ & 0.35075 & & & \\
& $(18)$ & 0.25509 & 12 & $(20,0)$ & 0.19642 \\
10 & & & & $(18,1)$ & 0.27826 \\
& $(14,2)$ & 0.38547 & & $(16,2)$ & 0.53549 \\
& $(12,3)$ & 0.22675 & & $(14,3)$ & 0.55410 \\
& $(10,4)$ & 0.56049 & & $(10,5)$ & 0.67444 \\
& $(85)$ & 0.40147 & & $(86)$ & 0.67203 \\
& $(66)$ & 0.49710 & & $(67)$ & 0.68661 \\
& $(47)$ & 0.42870 & & 0.67272 \\
& $(28)$ & 0.43649 & & & 0.66059 \\
\hline
\end{tabular}


limiting cases. Spectroscopic amplitudes for the weak coupling limit are given by eqs. (6) and (7). When the sum over $(\lambda \mu)$ is restricted to the single term with highest

TABLE 3

The $\alpha$-spectroscopic amplitudes for stripping to negative parity states of 1 hw excitation

\begin{tabular}{|c|c|c|c|c|c|c|c|}
\hline \multicolumn{8}{|c|}{ 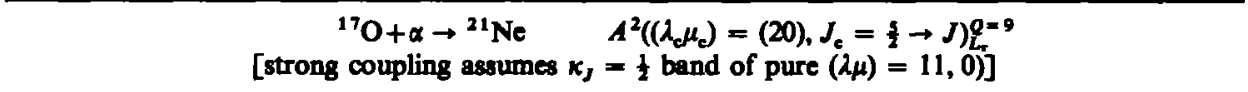 } \\
\hline$L_{\mathrm{r}}$ & $J$ & $A_{\text {mouk }}^{2}$ & $A_{\text {trons }}^{2}$ & $L_{r}$ & $\boldsymbol{J}$ & $A_{\text {wouk }}^{2}$ & $A_{\text {croos }}^{2}$ \\
\hline 1 & $\frac{3}{2}$ & 0.308 & 0.055 & 5 & $\frac{5}{2}$ & 0.324 & 0.027 \\
\hline 1 & $\frac{2}{3}$ & 0.335 & 0.004 & 5 & $\frac{7}{2}$ & 0.337 & 0.008 \\
\hline \multirow[t]{2}{*}{1} & $\frac{7}{2}$ & 0.303 & 0.059 & 5 & 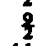 & 0.325 & 0.015 \\
\hline & & & & 5 & & 0.320 & 0.021 \\
\hline 3 & $\frac{1}{2}$ & 0.303 & 0.056 & 5 & $\frac{13}{3}$ & 0.338 & 0.017 \\
\hline 3 & $\frac{2}{\frac{3}{2}}$ & 0.332 & 0.022 & 5 & $\frac{2}{2}$ & 0.254 & 0.122 \\
\hline 3 & $\frac{1}{2}$ & 0.332 & 0.015 & & & & \\
\hline 3 & $\frac{7}{2}$ & 0.313 & 0.028 & 7 & $\frac{9}{2}$ & 0.330 & 0.012 \\
\hline 3 & $\frac{2}{2}$ & 0.338 & 0.011 & 7 & $\frac{11}{2}$ & 0.334 & 0.003 \\
\hline \multirow[t]{4}{*}{3} & 41 & 0.280 & 0.090 & 7 & $\frac{13}{3}$ & 0.325 & 0.012 \\
\hline & & & & 7 & $\frac{2}{15}$ & 0.329 & 0.015 \\
\hline & & $\therefore$ & & 7 & $\frac{17}{2}$ & 0.337 & 0.024 \\
\hline & & & & 7 & $\frac{19}{2}$ & 0.225 & 0.156 \\
\hline
\end{tabular}

${ }^{18} \mathrm{O}+\alpha \rightarrow{ }^{22} \mathrm{Ne} \quad A^{2}\left(\left(\lambda_{\mathrm{o}} \mu_{\mathrm{c}}\right)=(40), J_{\mathrm{c}}=0 \rightarrow J=L_{\mathrm{r}}\right)_{L_{r}}^{Q-9}$

[strong coupling assumes $x=1$ band of p $\quad(\lambda \mu)=(11,1)$ ]

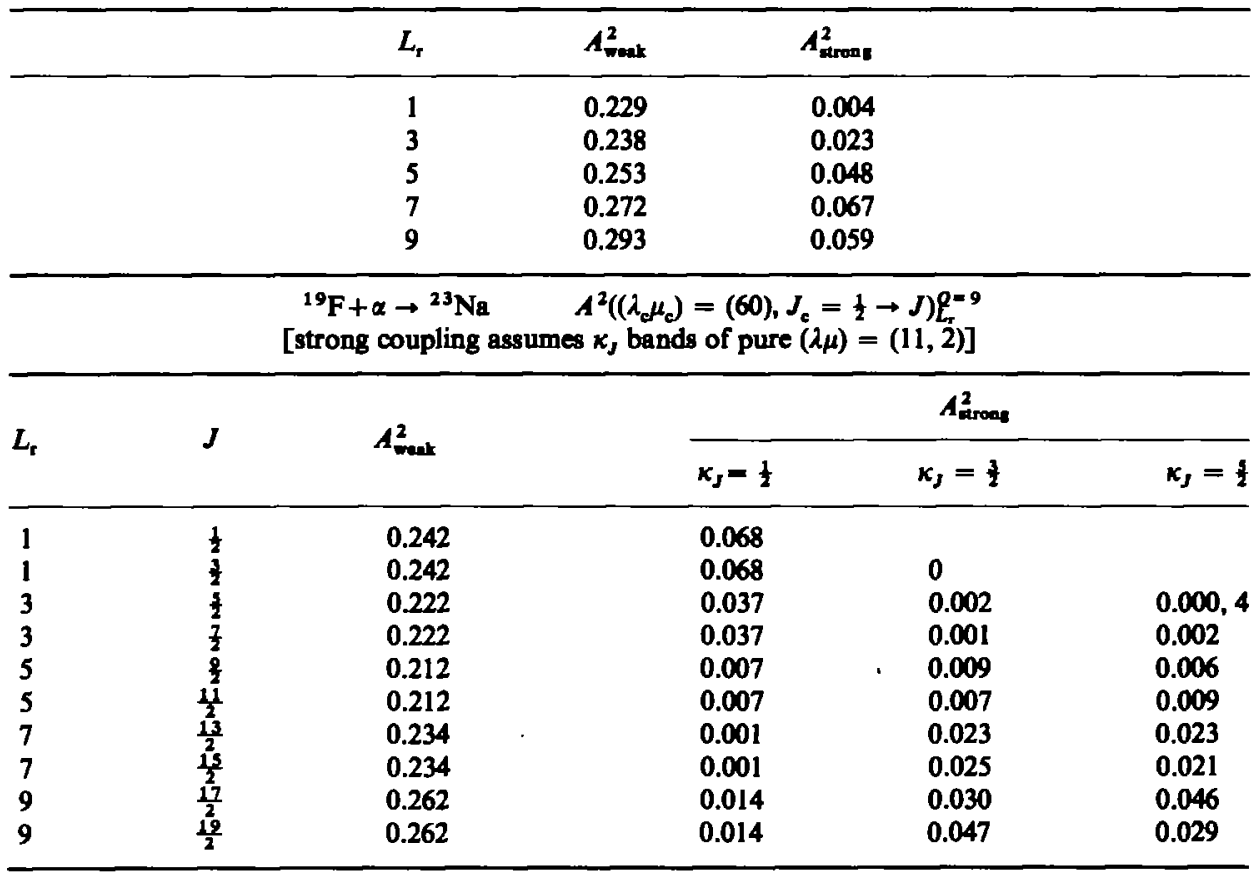


TABLE 3 (continued)

\begin{tabular}{|c|c|c|c|}
\hline \multicolumn{4}{|c|}{$\begin{array}{l}\left.{ }^{20} \mathrm{Ne}+\alpha \rightarrow{ }^{24} \mathrm{Mg} \quad A^{2}\left(\left(\lambda_{\mathrm{c}} \mu_{\mathrm{c}}\right)=(80), J_{\mathrm{c}}=0 \rightarrow J=L_{\mathrm{r}}\right)\right\}^{q=9} \\
\text { [strong coupling assumes } k \text { bands of pure }(\lambda \mu)=(11,3)]\end{array}$} \\
\hline \multirow[t]{2}{*}{$L_{\mathrm{r}}$} & \multirow[t]{2}{*}{$A_{\text {weak }}^{2}$} & \multicolumn{2}{|c|}{$A_{\text {mrone }}^{2}$} \\
\hline & & $x=1$ & $\kappa=3$ \\
\hline 1 & 0.153 & 0.016 & \\
\hline 3 & 0.266 & 0.063 & $0.000,2$ \\
\hline 5 & 0.203 & 0.059 & 0.005 \\
\hline 7 & 0.200 & 0.006 & 0.029 \\
\hline 9 & 0.230 & 0.028 & 0.089 \\
\hline
\end{tabular}

possible $(\lambda \mu)$, or when weighted with proper amplitudes for each $(\lambda \mu)$, these equations can also be used for $\mathrm{SU}(3)$ strong coupling or intermediate coupling wave functions. [For $S \neq 0$, the final states $\kappa L$ also have to be weighted with coefficients $c_{x L}^{x J}$; see eqs. (3.1) and (3.6) of ref. ${ }^{35}$ ).] Results for the strong and weak coupling limits are compared in table 3 for states of 1 hw excitation in light s-d shell nuclei. Since the weak coupling function corresponds most closely to an $\alpha$-particle moving about the core, spectroscopic factors for the weak coupling functions are in general an order of magnitude larger than those for the strong coupling limit. In the strong coupling limit there are marked $L_{\mathrm{r}}$ and $J$-dependences related to the fact that the $L_{\mathrm{c}}$ and $L_{\mathrm{r}}$ must "fit" into a state of definite $(\lambda \mu)$. In the weak coupling limit on the other hand there is very little $L_{\mathrm{r}}$ and $J$ dependence. In the limit in which the $N_{(\lambda \mu)}^{Q}$ can be replaced by an average value, the amplitudes are $L_{\mathrm{r}}$ independent. For high values of $Q$, it is thus sufficient to know an average value of $N_{(\lambda \mu)}^{Q}$. For $Q=12$ in ${ }^{24} \mathrm{Mg}$, for example, (table 2) the average value of $N$ is 0.548 ; the values of $A_{\text {weak }}^{2}$ are $0.56,0.56,0.55,0.55$, $0.57,0.58,0.60$ for $L_{\mathrm{r}}$ values of $0,2, \ldots, 12$, respectively. For $A=20-28$, relative spectroscopic factors for ground-state to ground-state transitions $(Q=8)$ are in surprisingly good agreement with the SU(3) strong coupling predictions ${ }^{36}$ ), with the exception of $A=24$ for which the experimental spectroscopic factor is too large by a factor of about two. In this case the predicted $0^{+} \rightarrow 0^{+}$weak to strong coupling ratio is 2.46 . The $0^{+}$weak coupling wave function in ${ }^{24} \mathrm{Mg}$, based on a $0^{+}(80){ }^{20} \mathrm{Ne}$ core, has $(\lambda \mu)=(84),(46)$ and (08) components of $40.6,34.7$ and $24.7 \%$. It is interesting to note that the shell-model calculation of Akiyama et al. ${ }^{53}$ ) predicts significant (46) and (08) admixtures for the ground state of ${ }^{24} \mathrm{Mg}$. The predicted shell model (80), (46) and (08) percentages are $72.8,11.0$ and $4.8 \%$, the missing percentage corresponding to states of lower space symmetry. The ground-state wave function for ${ }^{24} \mathrm{Mg}$ thus seems to be a superposition of strong and weak coupling cluster limits. In the extreme weak coupling limit, however, all of the strength for higher $L$-transfers would be shifted into the $\kappa=0$ band, whereas the experimentally observed ratios are much closer to the SU(3) strong coupling limit ${ }^{38}$ ). For pure (sd) $n$ configurations in light s-d shell nuclei, therefore, the SU(3) strong coupling model gives a very good approximation for $\alpha$-spectroscopic amplitudes. The analysis 
for negative parity core excited states is as yet not sufficiently complete to decide between the weak and strong coupling limits.

\section{The "Be clusters}

The cluster system, core $+2 \alpha$, has been treated by Katō and Bandō ${ }^{17}$ ) who have calculated the eigenvalues of the overlap operator $1-K$ for the ${ }^{16} \mathrm{O}+\alpha+\alpha$ cluster system by an elegant generating function technique. For nuclei with a core with $\left(\lambda_{\mathrm{c}} \mu_{\mathrm{c}}\right) \neq(00)$ the $\mathrm{SU}(3)$ recoupling techniques developed in sects. 2 and 3 may have some advantage. Although these techniques can be used for any three-cluster system for which $\mathrm{SU}(3)$ is a meaningful symmetry, recent experimental results for the $\left({ }^{12} \mathrm{C}, \alpha\right)$ and $\left({ }^{14} \mathrm{~N},{ }^{6} \mathrm{Li}\right)$ reactions $\left.{ }^{43-46}\right)$ make the core $+{ }^{8} \mathrm{Be}$ cluster of particular interest. For these reactions it is sufficient to treat a cluster model in which the $2 \alpha$ cluster is approximated by an eight-particle system corresponding to the $0^{+}, 2^{+}, 4^{+}$ states in ${ }^{8} \mathrm{Be}$ with wave functions belonging to the $\mathrm{SU}(3)$ representation $(40)$. The technique to be used is sufficiently general so that higher excitations of the relative motion of the $\alpha+\alpha$ system are easily included. In the numerical applications, however, the excitation of the internal degrees of freedom of the ${ }^{8} \mathrm{Be}$ system will be restricted to those which occur in the ${ }^{12} \mathrm{C}$ or ${ }^{14} \mathrm{~N}$ ground-state wave functions.

In the notation of sect. 2 and fig. $1 b$ the cluster wave function for a core $+\alpha+\alpha$ system can be written in SU(3) strong-coupled form as

$$
\begin{aligned}
& \Psi^{u}=\mathscr{A}\left[\phi_{A-8}\left(\xi_{c}\right)^{\left(\lambda_{0} \mu_{0}\right)\left(\gamma_{0} \mathrm{l}\right.} \times \mathscr{A}^{\prime}\left[\phi_{4}\left(\xi_{\alpha_{1}}\right) \phi_{4}\left(\xi_{\alpha_{2}}\right) \Phi^{(q 0)}\left(r_{\mathrm{rel}}^{\prime}\right)\right.\right.
\end{aligned}
$$

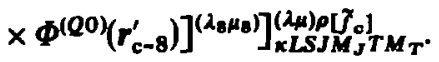

With both $\lambda_{c} \neq 0, \mu_{c} \neq 0$ the multiplicity label $\rho$ is needed for the SU(3) coupled function whenever the coupling of $\left(\lambda_{c} \mu_{c}\right) \times\left(\lambda_{8} \mu_{8}\right)$ yields the representation $(\lambda \mu)$ with a $d$-fold multiplicity with $d>1$. [The choice of $\rho(\rho=1, \ldots, d)$ follows refs. ${ }^{31,34}$ ).] The relative motion harmonic oscillator functions in eq. (31) are

$$
\Phi_{l m}^{(q 0)}\left(r_{\text {rel }}^{\prime}\right)=\Phi_{l m}^{(q 0)}\left(\left[\frac{4 \times 4 m \omega}{8 \hbar}\right]^{t}\left(R_{a_{1}}-R_{a_{2}}\right)\right)=\Phi_{l m}^{(q 0)}\left(\sqrt{\frac{1}{2}} R_{a_{1}}^{\prime}-\sqrt{\frac{1}{2}} R_{a_{2}}^{\prime}\right),
$$

where

$$
\begin{gathered}
R_{\alpha_{1}}^{\prime}=[4 m \omega / \hbar]^{\frac{1}{2}} \frac{1}{4}\left(r_{A}+r_{A-1}+r_{A-2}+r_{A-3}\right), \\
R_{\alpha_{2}}^{\prime}=[4 m \omega / \hbar]^{\frac{1}{2}} \frac{1}{4}\left(r_{A-4}+r_{A-5}+r_{A-6}+r_{A-7}\right), \\
\Phi_{L M}^{(Q 0)}\left(r_{c-8}^{\prime}\right)=\Phi_{L M}^{(Q 0)}\left(\left[\frac{(A-8) 8}{A} \frac{m \omega}{\hbar}\right]^{\frac{1}{2}}\left(\frac{r_{A}+\ldots+r_{A-7}}{8}-\frac{r_{A-8}+\ldots+r_{1}}{A-8}\right)\right) .
\end{gathered}
$$

The operator antisymmetrizes between the two $\alpha$-clusters. It annihilates states with $q<4$ since the eight-particle system must carry at least four oscillator quanta. The number of oscillator quanta in the relative motion function is thus restricted 
to $q \geqq 4$. The identity of the two $\alpha$-particles further restricts $q$ to even values, $q=4,6,8, \ldots$. The spectroscopic amplitudes for $\left({ }^{12} \mathrm{C} \rightarrow \alpha+8\right.$-particle cluster $)$ or $\left({ }^{14} \mathrm{~N} \rightarrow{ }^{6} \mathrm{Li}+8\right.$-particle cluster), calculated with harmonic oscillator shell-model wave functions, further restrict the internal eight-particle cluster function to the SU(3) representation (40). For our purposes therefore cluster states of the form of eq. (31), with $q$ restricted to $q=4$, will be sufficient. The formalism, however, will be carried out for general $q$. Even values of $q$, with $q>4$, correspond to the dissociation of nucleus $A$ into a residual nucleus $(A-8)$ and two dissociated $\alpha$ particles not in the ground-state (40) band of ${ }^{8} \mathrm{Be}$.

The method of calculation parallels that for single $\alpha$-clusters. It again involves two basic steps: (1) the transformation of overlaps of "cluster-like" functions to overlaps of true cluster functions, and (2) the calculation of the overlaps of cluster-like functions, which is carried out by projecting these onto properly antisymmetrized shell-model functions. The cluster-like function $\Psi^{w}$ is obtained from the true cluster function $\Psi^{\prime \prime}$ of eq. (31) by replacing the relative motion oscillator function $\Phi\left(r_{c-8}^{\prime}\right)$ in eq. (31) by an oscillator function $\Phi\left(\boldsymbol{R}_{8}^{\prime}=\sqrt{\frac{1}{2}}\left(\boldsymbol{R}_{\alpha_{1}}^{\prime}+\boldsymbol{R}_{\alpha_{2}}^{\prime}\right)\right.$ ) and by augmenting the internal function $\phi\left(\xi_{\text {core }}\right)$ by a wave function of 0 s excitation of the c.m. motion of the core nucleus:

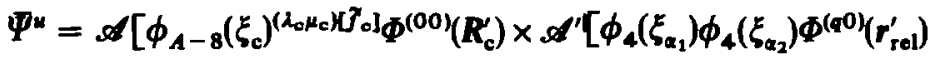

$$
\begin{aligned}
& \left.\left.\times \Phi^{(Q 0)}\left(R_{8}^{\prime}\right)\right]^{\left(\lambda_{g} \mu_{8}\right)}\right]^{(\lambda \mu) \rho\left[f_{c]}\right]} .
\end{aligned}
$$

The transformation from $\Psi^{u}$ to $\Psi^{u^{\prime}}$ is accomplished by the analogues of eqs. (15), (17) and (18) applied to the $2 \times 2$ unitary transformation:

$$
\begin{gathered}
R_{8}^{\prime}=\left[\frac{A-8}{A}\right]^{t} r_{\mathrm{c}-8}^{\prime}+\left[\frac{8}{A}\right]^{\frac{1}{2}} R_{\mathrm{c}, \mathrm{m} .}^{\prime}, \\
\boldsymbol{R}_{\mathrm{c}}^{\prime}=-\left[\frac{8}{A}\right]^{\frac{1}{r_{\mathrm{c}-8}^{\prime}}+\left[\frac{A-8}{A}\right]^{\frac{1}{t}} R_{\mathrm{c} . \mathrm{m} .}^{\prime} .}
\end{gathered}
$$

This yields, e.g.

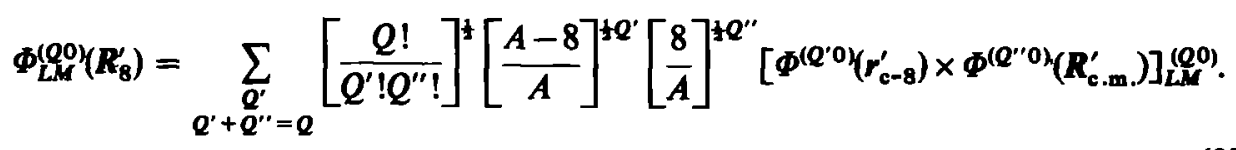

This transformation, together with two SU(3) Racah or 6-( $\lambda \mu)$ recoupling transformations, make it possible to expand the cluster-like function $\Psi^{m}$ for given $\left(\lambda_{c} \mu_{c}\right), q, Q,\left(\lambda_{8} \bar{\mu}_{8}\right)$, coupled to final $(\lambda \mu)$, in terms of true cluster functions with the 
same $\left(\lambda_{\mathrm{c}} \mu_{\mathrm{c}}\right), q$, but different $\left(\lambda_{8} \mu_{8}\right)$ and $Q^{\prime} \leqq Q$, coupled to resultant $\left(\lambda^{\prime} \mu^{\prime}\right)$ :

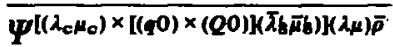

$$
\begin{aligned}
& =\sum_{\left(\lambda^{\prime} \mu^{\prime}\right) \rho} \sum_{\left(\lambda_{\theta} \mu_{8}\right)} \sum_{\substack{Q^{\prime \prime}=0 \\
Q^{\prime}+Q^{\prime \prime}=Q}}\left[\frac{Q !}{Q^{\prime} ! Q^{\prime \prime} !}\right]^{ \pm}\left[\frac{A-8}{A}\right]^{ \pm Q^{\prime}}\left[\frac{8}{A}\right]^{1 Q^{\prime \prime}} \\
& \times U\left((q 0)\left(Q^{\prime} 0\right)\left(\bar{\lambda}_{8} \bar{\mu}_{8}\right)\left(Q^{\prime \prime} 0\right) ;\left(\lambda_{8} \mu_{8}\right)(Q 0)\right) U\left(\left(\lambda_{c} \mu_{c}\right)\left(\lambda_{8} \mu_{8}\right)(\lambda \mu)\left(Q^{\prime \prime} 0\right) ;\left(\lambda^{\prime} \mu^{\prime}\right) \rho_{-} ;\left(\bar{\lambda}_{8} \bar{\mu}_{8}\right)_{-} \bar{\rho}\right) \\
& \times\left[\mathscr { A } \left[\phi^{\left(\lambda_{0} \mu_{c}\right)} \tilde{J}_{0}^{\prime} \cdot\left(\xi_{\mathrm{c}}\right) \times \mathscr{A}^{\prime}\left[\phi\left(\xi_{a_{1}}\right) \phi\left(\xi_{a_{2}}\right) \Phi^{(\mathrm{q} 0)}\left(r_{\mathrm{rel}}^{\prime}\right)\right.\right.\right. \\
& \left.\left.\left.\times \Phi^{\left(Q^{\prime} 0\right)}\left(r_{\mathrm{c}-8}^{\prime}\right)\right]^{\left(\lambda_{8} \mu_{8}\right)}\right]^{\left(\lambda^{\prime} \mu^{\prime}\right) \rho} \times \Phi^{\left(Q^{\prime \prime} 0\right)}\left(R_{\text {c.m. }}^{\prime}\right)\right]^{(\lambda \mu)\left[\gamma_{0}\right]} \text {. }
\end{aligned}
$$

In the simple case when all cluster states are based on an $(A-8)$-particle core with a single $\left(\lambda_{\mathrm{c}} \mu_{\mathrm{c}}\right)$, and the eight-particle cluster has a single, fixed value of $q(q=4$ in our applications), the overlap of cluster-like functions $\Psi$ is thus related to the overlap of the true cluster functions $\Psi$ by

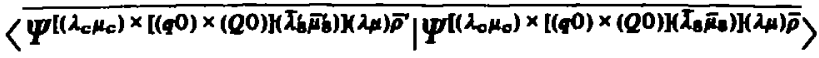

$$
\begin{aligned}
& =\left[\frac{A-8}{A}\right]^{Q}\left\langle\Psi^{\left[\left(\lambda_{c} \mu_{0}\right) \times[(q 0) \times(Q 0)]\left(\lambda_{k} \bar{\mu}_{k}\right)\right]\left(\lambda_{\mu}\right) \bar{p}^{\prime}} \mid \Psi\left[\left(\lambda_{\sigma} \mu_{c}\right) \times[(q 0) \times(Q 0)]\left(\bar{\lambda}_{8} \bar{\mu}_{8}\right)\right\}\left(\lambda_{\mu}\right) \bar{\rho}\right\rangle
\end{aligned}
$$

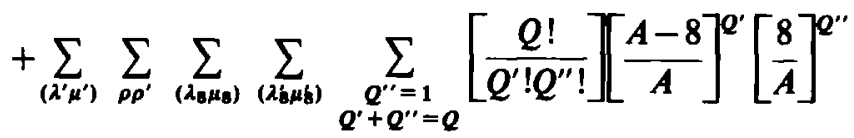

$$
\begin{aligned}
& \times U\left((q 0)\left(Q^{\prime} 0\right)\left(\bar{\lambda}_{8}^{\prime} \bar{\mu}_{8}^{\prime}\right)\left(Q^{\prime \prime} 0\right) ;\left(\lambda_{8}^{\prime} \mu_{8}^{\prime}\right)(Q 0)\right) U\left(\left(\lambda_{c} \mu_{c}\right)\left(\lambda_{8}^{\prime} \mu_{8}^{\prime}\right)(\lambda \mu)\left(Q^{\prime \prime} 0\right) ;\left(\lambda^{\prime} \mu^{\prime}\right) \rho_{-}^{\prime} ;\left(\bar{\lambda}_{8}^{\prime} \bar{\mu}_{8}^{\prime}\right)_{-} \bar{\rho}^{\prime}\right) \\
& \left.\times U\left((q 0)\left(Q^{\prime}\right)\right)\left(\lambda_{8} \bar{\mu}_{8}\right)\left(Q^{\prime \prime} 0\right) ;\left(\lambda_{8} \mu_{8}\right)(Q 0)\right) U\left(\left(\lambda_{c} \mu_{\mathrm{c}}\right)\left(\lambda_{8} \mu_{8}\right)(\lambda \mu)\left(Q^{\prime \prime} 0\right) ;\left(\lambda^{\prime} \mu^{\prime}\right) \rho_{-} ;\left(\bar{\lambda}_{8} \bar{\mu}_{8}\right)-\bar{\rho}\right) \\
& \times\left\langle\Psi^{\left.\prime\left(\lambda_{c} \mu_{c}\right) \times\left[(g 0) \times\left(Q^{\prime} 0\right)\right\}\left(\lambda \lambda_{b}\right)\right]\left(\lambda^{\prime} \mu^{\prime}\right) \rho^{\prime}} \mid \Psi^{\left[\left(\lambda_{c} \mu_{0}\right) \times\left[(q 0) \times\left(Q^{\prime} 0\right)\right\}\left(\lambda_{B} \mu_{B}\right)\right]\left(\lambda^{\prime} \mu^{\prime}\right) \rho}\right\rangle .
\end{aligned}
$$

The overlaps of two true cluster states $\Psi$ with different $\left(\lambda_{8} \mu_{8}\right)$ and $\left(\lambda_{8}^{\prime} \mu_{8}^{\prime}\right)$ but the same $\left(\lambda_{\mathrm{c}} \mu_{\mathrm{c}}\right), q$, and hence $Q$, can thus be calculated if the overlaps for cluster states with $Q^{\prime}<Q$ are known from an earlier step in a chain calculation. For $Q^{\prime}=Q-1$, the representations $\left(\lambda^{\prime} \mu^{\prime}\right)$ in the last term of eq. (39) are those for which a removal of a single square from the Young tableau for $(\lambda \mu)$ leads to a permissible tableau with a Pauli-allowed cluster function; for $Q^{\prime}=Q-2$, the representations $\left(\lambda^{\prime} \mu^{\prime}\right)$ are those for which the removal of two symmetrically coupled squares from the Young tableau for $(\lambda \mu)$ leads to a permissible cluster function; etc. The derivation of eq. (39) has made use of the orthogonality of c.m. excitation functions with different $Q^{\prime \prime}$ and of the orthogonality of cluster functions with different $\left(\lambda^{\prime} \mu^{\prime}\right)$.

In order to expand the cluster-like functions $\Psi$ in terms of shell-model functions in step (2) of the calculation process, it will be useful to apply the transformation (15) 
to the $2 \times 2$ unitary transformation,

$$
r_{\text {rel }}^{\prime}=\sqrt{\frac{1}{2}} R_{\alpha_{1}}^{\prime}-\sqrt{\frac{1}{2}} R_{\alpha_{2}}^{\prime}, \quad R_{8}^{\prime}=\sqrt{\frac{1}{2}} R_{\alpha_{1}}^{\prime}+\sqrt{\frac{1}{2}} R_{\alpha_{2}}^{\prime},
$$

to expand $\Phi^{\left(Q^{(0)}\right)}\left(r_{\text {reil }}^{\prime}\right)$ and $\Phi^{(Q 0)}\left(R_{8}^{\prime}\right)$ in terms of the $\alpha$-cluster functions $\Phi^{\left(Q_{1}{ }^{0}\right)}\left(R_{z_{1}}^{\prime}\right)$, $\Phi^{\left(Q_{2}\right)}\left(R_{\alpha_{2}}^{\prime}\right)$. Simple SU(3) recoupling leads to

$$
\begin{aligned}
& {\left[\Phi^{(q 0)}\left(r_{\mathrm{ret}}^{\prime}\right) \times \Phi^{(Q 0)}\left(R_{8}^{\prime}\right)\right]^{\left(\lambda_{8} \mu_{8}\right)}=\sum_{\substack{Q_{2} \\
Q_{1}+Q_{2}=Q+q}}\left[\Phi^{\left(Q_{1} 0\right)}\left(R_{\alpha_{1}}^{\prime}\right) \times \Phi^{\left(Q_{2} 0\right)}\left(R_{\alpha_{2}}\right)\right]^{\left(\lambda_{8} \mu_{8}\right)} } \\
& \times \sum_{\substack{q_{2} \\
q_{1}+q_{2}=q}} X_{\mathrm{SU}(3)}\left[\begin{array}{ccc}
\left(q_{1} 0\right) & \left(q_{2} 0\right) & (q 0) \\
\left(Q_{1}-q_{1}, 0\right) & \left(Q_{2}-q_{2}, 0\right) & (Q 0) \\
\left(Q_{1} 0\right) & \left(Q_{2} 0\right) & \left(\lambda_{8} \mu_{8}\right)
\end{array}\right](-1)^{q_{2}} \\
& \times\left[\left(\begin{array}{l}
Q_{1} \\
q_{1}
\end{array}\right)\left(\begin{array}{l}
Q_{2} \\
q_{2}
\end{array}\right)\left(\begin{array}{c}
q \\
q \\
q_{1}
\end{array}\right)\left(\begin{array}{c}
Q \\
Q_{1}-q_{1}
\end{array}\right)\right]^{\frac{1}{2}} .
\end{aligned}
$$

The derivation of eq. (41) uses the fact that the coupling of two polynomials in the same harmonic oscillator creation operators contributes a normalization factor ${ }^{51}$ )

$$
\left[P^{\left(q_{1}\right)}\left(\eta_{i}\right) \times P^{\left(Q_{1}-q_{1}, 0\right)}\left(\eta_{i}\right)\right]^{\left(Q_{1} 0\right)} \ldots \equiv\left[\left(\begin{array}{l}
Q_{1} \\
q_{1}
\end{array}\right)\right]^{z} P^{\left(Q_{1} 0\right)}\left(\eta_{i}\right) .
$$

The symbol in square brackets in eq. (41) is an SU(3) 9-( $(\lambda \mu)$ recoupling coefficient in unitary form ${ }^{34}$ ). Since it involves only SU(3) representations corresponding to one- or two-rowed tableaux, it is equivalent to an ordinary $9 j$ transformation coefficient (in unitary form) which is of a particularly simple form since it contains four "stretched" couplings. The transformation properties of the Moshinsky brackets under the group $\mathrm{U}(2) \times \mathrm{U}(3)$, as elucidated by Brody and Moshinsky ${ }^{36}$ ) and Kaufman and Noack ${ }^{57}$ ) show that the transformation coefficient in eq. (41) can also be expressed as a simple $\mathrm{SU}(2) D$-function with Euler angles $\alpha, \beta, \gamma=0$, $\frac{1}{2} \pi, 0$; so that

$$
\begin{aligned}
& {\left[\Phi^{(90)}\left(r_{\text {rel }}^{\prime}\right) \times \Phi^{(\mathbf{Q} 0)}\left(R_{8}^{\prime}\right)\right]_{\ldots}^{\left(\lambda_{8} \mu_{8}\right)}=\sum_{\substack{Q_{2} \\
Q_{1}+Q_{2}=Q+q}}\left[\Phi^{\left(Q_{1} 0\right)}\left(R_{a_{1}}^{\prime}\right) \times \Phi^{\left(Q_{2} 0\right)}\left(R_{\alpha_{2}}^{\prime}\right)\right]_{\ldots}^{\left(\lambda_{8} \mu_{8}\right)}}
\end{aligned}
$$

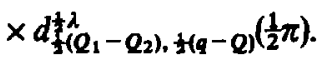

The calculation of the overlaps of cluster-like functions $\left\langle\Psi^{*} \mid \Psi^{\text {D }}\right\rangle$ through projection onto shell-model states, eq. (9), is thus reduced to the calculation of $\left\langle\Psi_{\mathrm{SM}}^{y} \mid \bar{\Psi}^{\prime}\right\rangle$ where the double-barred $\bar{\Psi}$ are double- $\alpha$ cluster-like functions

$$
\begin{aligned}
& \Psi=\mathscr{A}\left[\phi^{\left.\left(\lambda_{c} \mu_{0}\right)\right]_{c} \gamma^{\prime}}\left(\xi_{c}\right) \Phi^{(00)}\left(R_{c}^{\prime}\right)\right. \\
& \left.\times \mathscr{A}^{\prime}\left[\phi\left(\xi_{\alpha_{1}}\right) \phi\left(\xi_{\alpha_{2}}\right) \Phi^{\left(Q_{1} 0\right.}\left(R_{\left.\alpha_{1}\right)}^{\prime}\right) \Phi^{\left(Q_{2} 0\right)}\left(R_{\alpha_{2}}^{\prime}\right)\right]^{\left(\lambda_{8} \mu_{8}\right)}\right]_{\ldots}^{\left(\lambda_{\mu}\right) \rho\left[\gamma_{0}\right]} .
\end{aligned}
$$

The cluster system ${ }^{12} \mathrm{C}+{ }^{8} \mathrm{Be}$ has been chosen as the prime example. The nucleus 
${ }^{20} \mathrm{Ne}$ is of particular interest since it has been investigated both through the $\left({ }^{12} \mathrm{C}, \alpha\right)$ reaction $\left.{ }^{43}\right)$ and the $\left({ }^{14} \mathrm{~N},{ }^{6} \mathrm{Li}\right)$ reaction $\left.{ }^{44,45}\right)$ on ${ }^{12} \mathrm{C}$. These reactions excite a number of bands that are not observed in four-nucleon stripping reactions on ${ }^{16} \mathrm{O}$. The $K=0^{+}$band with bandhead at $7.20 \mathrm{MeV}$ has been tentatively assigned as a band based on the $8 p-4 h$ configuration in ${ }^{20} \mathrm{Ne}$, with $\mathrm{SU}(3)$ representations (84) and (04) for the $8 \mathrm{p}$ and $4 \mathrm{~h}$ pieces, respectively. The coupling of these representations leads to a large number of resultant $(\lambda \mu)$. The $S U(3)$ symmetry $(\lambda \mu)=(88)$, however, corresponds to the most strongly deformed intrinsic state of ${ }^{20} \mathrm{Ne}$. Since other lowlying rotational bands based on core-excited states in ${ }^{20} \mathrm{Ne}$ seem to be approximated well by $\mathrm{SU}(3)$ strong-coupled wave functions corresponding to large intrinsic deformations, the ${ }^{8} \mathrm{Be}$ cluster calculations will be limited to the SU(3) strongcoupled approximation, based on states corresponding to large intrinsic deformations. Besides the $K=0^{+}$band at $7.20 \mathrm{MeV}$, the $K=2^{-}$band (bandhead $4.97 \mathrm{MeV}$ ) with $(\lambda \mu)=(82), 1 \hbar \omega$ excitation, and the $K=0^{+}$band (bandhead $6.72 \mathrm{MeV}$ ), predominantly $(\lambda \mu)=(42), 0 \hbar \omega$ excitation, are of particular interest since they are observed in eight-nucleon transfer reactions but have $\alpha$-spectroscopic factors of zero in the extreme $S U(3)$ limit. The ground-state $K=0^{+}$band, $(\lambda \mu)=(80)$, and the 5.80 $\mathrm{MeV} K=0^{-}$band, $(\lambda \mu)=(90)$, are populated strongly both in $\alpha$ and ${ }^{8} \mathrm{Be}$ transfer stripping reactions. Additional states of $2 \hbar \omega$ excitation might be expected ${ }^{58,59}$ ) around $10 \mathrm{MeV}$. For states of $2 \hbar \omega$ excitation the SU(3) symmetries (84) and (92) correspond to the largest intrinsic deformations. All of the ${ }^{20} \mathrm{Ne}$ bands listed can be expected to have significant overlaps with $\left({ }^{12} \mathrm{C}+{ }^{8} \mathrm{Be}\right)$ cluster wave functions of the form of eq. (31), based on a ${ }^{12} \mathrm{C}$ core with $\left(\lambda_{\mathrm{c}} \mu_{\mathrm{c}}\right)=(04)$, with $q=4$, and $Q$ ranging from $Q=8$ ( $0 h \omega$ excitation) to $Q=12$ ( $4 \hbar \omega$ excitation).

The general complexity of the calculations can be illustrated by the special example $Q=9$ (excitations of $1 h \omega$ in ${ }^{20} \mathrm{Ne}$ ). With $q=4, Q=9$, the possible $\left(\lambda_{8} \mu_{8}\right)$ are $(13,0),(11,1),(92),(73),(54)$. All of these can couple with $\left(\lambda_{c} \mu_{c}\right)=(04)$ to make the single allowed nonspurious state with $(\lambda \mu)=(90)$ in ${ }^{20} \mathrm{Ne}$. For $(\lambda \mu)=(90)$ the overlap matrix $\left\langle\Psi^{u} \mid \Psi^{\nu}\right\rangle$ is thus a $5 \times 5$ matrix with four eigenvalues of zero and a single nonzero eigenvalue. All of the possible $\left(\lambda_{8} \mu_{8}\right)$ except $(13,0)$ can couple with $\left(\lambda_{\mathrm{c}} \mu_{\mathrm{c}}\right)=(04)$ to make the Pauli-allowed states with $(\lambda \mu)=(82),(71)$. For both of these the overlap matrices are $4 \times 4$, again with a single nonzero eigenvalue. For $(\lambda \mu)=(63),(52)$ the overlap matrices are $3 \times 3$, etc. ... The double- $\alpha$ cluster-like functions $\bar{\Psi}$, eq. (44), which survive the antisymmetrization process are restricted to the $Q_{1} Q_{2}$ (or $Q_{2} Q_{1}$ ) values: 94,85 and 76 . These can project onto only two types of shell-model states:

$$
\Psi_{\mathrm{SM}}=\Psi\left(\mathrm{s}^{4}\left[\mathrm{p}^{11}(01)\left[1^{3}\right](\mathrm{sd})^{5}\left(\lambda_{5} \mu_{5}\right)\left[f_{5}\right]=[1]\right]^{\left(\lambda_{\mu}\right)(0)}\right),
$$

with $\left(\lambda_{s} \mu_{5}\right)=(81),(62),(43), \ldots$, and

$$
\Psi_{\text {sM }}=\Psi\left(\mathrm{s}^{4} \mathrm{p}^{12}\left[(\mathrm{sd})^{3}\left(\lambda_{3} \mu_{3}\right)\left[1^{3}\right](\mathrm{pf})^{1}(30)[1]\right]_{\ldots}^{(2 \mu)(0]},\right.
$$

with $\left(\lambda_{3} \mu_{3}\right)=(60),(22)$. 
Two examples will suffice to illustrate the complexity of the recoupling process needed to calculate $\left\langle\Psi_{\mathrm{su}} \mid \Psi\right\rangle$. The function $\bar{\Psi}$ with $Q_{1} Q_{2}=49$ is the simplest example. It has a nonzero overlap only with the second type of shell-model function, with $\left(\lambda_{3} \mu_{3}\right)=(60)$, provided $(\lambda \mu)=(90)$. This overlap has the simple value

$\left[\frac{20 !}{4 ! 8 ! 4 ! 4 !}\right]^{\ddagger}\left[\frac{4 ! 12 ! 3 ! 1 !}{20 !}\right]^{\sharp}\left\{\left[\frac{4 !}{1 ! 1 ! 1 ! 1 !}\right]^{\ddagger} \frac{1}{2^{4}}\left[\frac{9 !}{2 ! 2 ! 2 ! 3 !}\right]^{\ddagger} \frac{1}{2^{9}} 4\right\}$

$\times U\left((04)(40)(\lambda \mu)(90) ;(00)\left(\lambda_{8} \mu_{8}\right)\right)\left[\frac{1}{33}\right]^{\frac{1}{2}}$.

The first factor comes from the action of antisymmetrizers $\mathscr{A}, \mathscr{A}^{\prime}, \ldots$ to the left. The second factor is the normalization factor associated with the decomposition of the antisymmetric shell-model wave function into the configuration $\mathrm{s}^{4} \mathrm{p}^{12} \mathrm{sd}^{3} \mathrm{pf}^{1}$. The factor in curly brackets is the transformation coefficient of eq. (15) for the decomposition of $\Phi^{(40)}\left(R_{\alpha_{1}}^{\prime}\right)$ and $\Phi^{(90)}\left(R_{a_{2}}^{\prime}\right)$ into single particle functions, the final factor of four coming from the four possible decompositions of $Q_{2}=9$ into $q_{1} q_{2} q_{3} q_{4}=2223,2232,2322,3222$. The $\mathrm{SU}(3)$ recoupling $U$-coefficient is needed to transform the SU(3) coupling from the order $\left[\mathrm{p}^{8}\left[\mathrm{p}^{4}\left[\mathrm{sd}^{3} \mathrm{pf}^{1}\right]\right]\right]$ to $\left[\left[\mathrm{p}^{8} \mathrm{p}^{4}\right]\left[\mathrm{sd}^{3} \mathrm{pf}^{1}\right]\right]$. With one representation of $(00)$ it is given by simple SU(3) dimensional factors and is zero unless $(\lambda \mu)=(90)$. The final factor is the p-shell $8+4$ particle c.f.p., given by eq. (27). The sd shell c.f.p. is trivially equal to unity.

The function $\bar{\Psi}$ with $Q_{1} Q_{2}=58$ has nonzero overlap with shell-model functions of both types, eqs. (45a) and (45b). The overlap with $\Psi_{s M}\left(s^{4} p^{12} s d^{3} \mathrm{pf}^{1}\right)$ is given by

$$
\begin{aligned}
& {\left[\frac{20 !}{4 ! 8 ! 4 ! 4 !}\right]^{\ddagger}\left[\frac{4 ! 12 ! 3 ! 1 !}{20 !}\right]^{\ddagger}\left\{\left[\frac{5 !}{1 ! 1 ! 1 ! 2 !}\right]^{\ddagger} \frac{4}{2^{5}}\left[\frac{8 !}{1 ! 2 ! 2 ! 3 !}\right]^{\frac{1}{2}} \frac{12}{2^{8}}\right\}}
\end{aligned}
$$

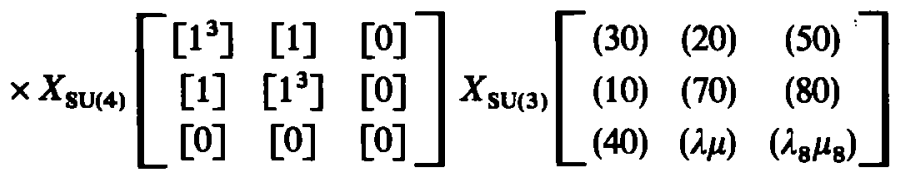

$$
\begin{aligned}
& \times U\left((20)(40)(\lambda \mu)(30) ;\left(\lambda_{3} \mu_{3}\right)(70)\right) \\
& \times U\left((04)(40)(\lambda \mu)(\lambda \mu) ;(00)\left(\lambda_{8} \mu_{8}\right)\right)\left[\frac{1}{33}\right]^{ \pm}\left\{\delta_{\left(\lambda_{3} \mu_{3}\right)(60)}+\frac{2}{3} \delta_{\left(\lambda_{3} \mu_{3}\right)(22)}\right\}(-1) \text {. }
\end{aligned}
$$

Additional recoupling transformations and coefficients are now needed to bring the final single particle wave functions into the order $\mathrm{s}^{4} \mathrm{p}^{12} \mathrm{sd}^{3} \mathrm{pf}^{1}$. The first of these, given by the two $X$-coefficients, is a 9-( $\lambda \mu)$, [ $]$ ] recoupling transformation used to transform the SU(3), SU(4) coupling for particles $13,14, \ldots, 20$ from the order $\left[\left[\mathrm{p}^{3}(\mathrm{sd})^{1}\right]\left[\mathrm{p}^{1}\left[(\mathrm{sd})^{2}(\mathrm{pf})^{1}\right]\right]\right]$ to $\left[\left[\mathrm{p}^{3} \mathrm{p}^{1}\right]\left[(\mathrm{sd})^{1}\left[(\mathrm{sd})^{2}(\mathrm{pf})^{1}\right]\right]\right]$. This is followed by recoupling transformations from $\left[(\mathrm{sd})^{1}\left[(\mathrm{sd})^{2}(\mathrm{pf})^{1}\right]\right]$ to $\left[\left[(\mathrm{sd})^{1}(\mathrm{sd})^{2}\right](\mathrm{pf})^{1}\right]$, a pure SU(3) 6-( $\lambda \mu$ ) transformation since the resultant function must be an SU(4) scalar; and finally a recoupling transformation from $\left[\mathrm{s}^{4}\left[\mathrm{p}^{8}\left[\mathrm{p}^{4}\left[(\mathrm{sd})^{3}(\mathrm{pf})^{1}\right]\right]\right]\right]$ to $\left[\mathrm{s}^{4}\left[\left[\mathrm{p}^{8} \mathrm{p}^{4}\right]\right.\right.$ $\left.\left.\left[(\mathrm{sd})^{3}(\mathrm{pf})^{1}\right]\right]\right]$, another pure $\mathrm{SU}(3) 6-(\lambda \mu)$ transformation. The final factor of $(-1)$ is also associated with this reordering process: It is convenient to sandwich the 
identity operator $\equiv P^{2}$ between $\Psi_{\mathrm{SM}}$ and $\bar{\Psi}$ in $\left\langle\Psi_{\mathrm{SM}} \mid \bar{\Psi}\right\rangle$, where $P$ is here the permutation operator which exchanges particles 16 and 17 . This $P$-operator is needed to bring particle numbers $13, \ldots 20$ to cardinal order, acting to the right after the recoupling process. It gives the factor $(-1)$ when acting on $\Psi_{\mathrm{sM}}$ to the left. The first recoupling coefficient in square brackets is the SU(4) recoupling coefficient ${ }^{34}$ ), needed for the first recoupling transformation. It is given by SU(4) dimensional factors and has the value $\frac{1}{4}$, (independent of phase conventions). The second recoupling coefficient in square brackets is the corresponding $S U(3)$ 9-( $(\lambda \mu)$ recoupling coefficient ${ }^{34}$ ). Since it contains SU(3) representations with one and two-rowed tableaux it can be read from tables of $S U(2) 9 j$ coefficients.

The overlap of $\bar{\Psi}\left(Q_{1} Q_{2}=58\right)$ with the shell-model function $\Psi\left(\mathrm{s}^{4} \mathrm{p}^{11} \mathrm{sd}^{5}\right)$ is given by

$$
\begin{aligned}
& {\left[\frac{20 !}{4 ! 8 ! 4 ! 4 !}\right]^{\frac{1}{2}}\left[\frac{4 ! 11 ! 5 !}{20 !}\right]^{\frac{1}{4}}\left\{\left[\frac{5 !}{1 ! 1 ! 1 ! 2 !}\right]^{\frac{1}{4}} \frac{4}{2^{5}}\left[\frac{8 !}{2 ! 2 ! 2 ! 2 !}\right]^{\frac{1}{2}} \frac{1}{2^{8}}\right\}} \\
& \quad \times U\left((30)(20)\left(\lambda_{8} \mu_{8}\right)(80) ;(50)\left(\lambda_{5} \mu_{5}\right)\right) U\left((04)(30)(\lambda \mu)\left(\lambda_{5} \mu_{5}\right) ;(01)\left(\lambda_{8} \mu_{8}\right)\right) \\
& \quad \times\left[\frac{1}{33}\right]^{\frac{1}{2}}\left\{-\frac{1}{2} \delta_{\left(\lambda_{5} \mu_{5}\right)(81)}+\left[\frac{27}{140}\right]^{\frac{1}{5}} \delta_{\left(\lambda_{5} \mu_{5}\right)(62)}\right\} .
\end{aligned}
$$

The overlaps $\left\langle\Psi_{\text {sM }} \mid \bar{\Psi}\right\rangle$ together with the transformation of eq. (41) lead to the overlaps of the cluster-like states $\left\langle\Psi^{\|} \mid \Psi^{\eta}\right\rangle$.

The quantum numbers within $u$ are identical to those within $v$ with the exception of $\left(\lambda_{8} \mu_{8}\right)$ so that the rows and columns of the overlap matrices can be labeled by $\left(\lambda_{8} \mu_{8}\right)$. With $\left(\lambda_{c} \mu_{c}\right)=(04)$ multiplicity labels $\rho$ are never needed. The final step in the calculation involves the relation between $\left\langle\Psi^{n} \mid \Psi^{v}\right\rangle$ and $\left\langle\Psi^{n} \mid \Psi^{v}\right\rangle$ given by eq. (39). With $q=4, Q=9$, and $(\lambda \mu)=(82)$, for example, only the first term with $Q^{\prime \prime}=0$ survives in eq. (39), since the single ${ }^{20} \mathrm{Ne}$ shell-model state with 1 h $\omega$ excitation and $(\lambda \mu)=(82)$ is free of spurious c.m. excitation. In this case the true cluster function and barred function overlaps differ only by the trivial factor $(20 / 12)^{9}$. With $(\lambda \mu)=(90)$, however, eq. (39) involves contributions from the $Q^{\prime \prime}=1$ term with $\left(\lambda^{\prime} \mu^{\prime}\right)=(80)$. With $\left(\bar{\lambda}_{8}^{\prime} \bar{\mu}_{8}^{\prime}\right)=(73),\left(\bar{\lambda}_{8} \bar{\mu}_{8}\right)=(54)$, e.g. the $Q^{\prime}=8$ overlaps with $\left(\lambda_{8}^{\prime} \mu_{8}^{\prime}\right)=(82),(63)$ and $\left(\lambda_{8} \mu_{8}\right)=(63),(44)$ contribute to the final value of the overlap of the true cluster functions.

The states of interest in ${ }^{20} \mathrm{Ne}$ all lead to overlap matrices $\left\langle\Psi^{*} \mid \Psi^{\nu}\right\rangle$ with a single nonzero eigenvalue, hence a single nonspurious eigenvector. These matrices have the simple (factored) form

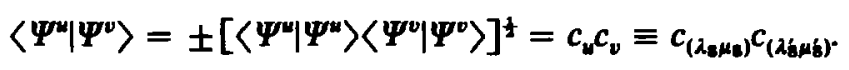

With $\left(\lambda_{c} \mu_{c}\right)=(04), q=4$, fixed $Q$ and $(\lambda \mu)$, the states $\Psi^{\prime \prime}$ are characterized by $\left(\lambda_{\mathrm{g}} \mu_{\mathrm{g}}\right)$. The eigenvector corresponding to the single nonzero eigenvalue has the simple form

$$
N \sum_{\left(\lambda_{8} \mu_{8}\right)} c_{\left(\lambda_{8} \mu_{8}\right)} \Psi\left(\left[(04) \times[(40) \times(Q 0)]\left(\lambda_{8} \mu_{8}\right)\right](\lambda \mu)\right),
$$


TABle 4

Be cluster states in ${ }^{20} \mathrm{Ne}$

\begin{tabular}{|c|c|c|c|c|}
\hline $\boldsymbol{Q}$ & $(\lambda \mu)$ & $\left(\lambda_{\theta} \mu_{8}\right)$ & {$\left[\left\langle\Psi^{\prime} \mid \Psi^{\prime}\right\rangle /(20 / 12)^{Q}\right]$} & $\left.\left.c_{\left(\lambda_{a(s)}\right)}\right)^{\prime}\right)$ \\
\hline 8 & (80) & $\begin{array}{l}(12,0) \\
(10,1) \\
(82) \\
(63) \\
(44)\end{array}$ & $\begin{array}{l}3 \times 7^{2} \times 13 / 2^{22} \\
3 \times 7 \times 11 \times 13 / 2^{20} \\
3^{5} \times 47^{2} / 7 \times 2^{23} \\
3 \times 11 \times 61^{2} / 2^{23} \\
3 \times 5 / 2^{8} \times 7\end{array}$ & $\begin{aligned} & 0.164701 \\
& 0.412926 \\
& 0.737738 \\
& 0.933548 \\
& 0.705946 \\
& 1 / N_{(80)}= 2.112\end{aligned}$ \\
\hline 8 & (42) & $\begin{array}{l}(82) \\
(63) \\
(44)\end{array}$ & $\begin{array}{l}3^{6} \times 5 \times 11 / 2^{23} \times 7 \\
3^{5} \times 5 / 2^{21} \\
3^{4} \times 5^{2} / 2^{15} \times 7\end{array}$ & $\begin{aligned} & 0.201626 \\
& 0.185724 \\
& 0.724993 \\
1 / N_{(42)}= & 0.601\end{aligned}$ \\
\hline 9 & (90) & $\begin{array}{l}(13,0) \\
(11,1) \\
(92) \\
(73) \\
(54)\end{array}$ & $\begin{array}{l}3^{5} \times 7^{2} / 11 \times 2^{20} \\
3^{5} \times 7^{2} \times 23^{2} / 5 \times 11 \times 2^{25} \\
3^{3} \times 7 \times 13 \times 37^{2} / 11 \times 2^{26} \\
3^{3}(313)^{2} / 5 \times 7 \times 11 \times 2^{22} \\
3^{9} / 7 \times 2^{26}\end{array}$ & $\begin{aligned} 0.320055 \\
0.581959 \\
0.672415 \\
0.403167 \\
-0.064480 \\
1 / N_{(90)}=1.060\end{aligned}$ \\
\hline 9 & (82) & $\begin{array}{l}(11,1) \\
(92) \\
(73) \\
(54)\end{array}$ & $\begin{array}{l}3^{2} \times 5^{2} \times 7^{2} \times 13 / 11 \times 2^{26} \\
3 \times 5^{2} \times 7^{4} \times 13 / 11 \times 2^{27} \\
3 \times 5^{2} \times 13 \times 17^{2} / 7 \times 2^{23} \\
3^{2} \times 5^{5} \times 13^{2} / 7 \times 2^{27}\end{array}$ & $\begin{aligned} & 0.138801 \\
& 0.396658 \\
& 0.690044 \\
& 0.708525 \\
1 / N_{(82)}= & 1.155\end{aligned}$ \\
\hline 9 & (71) & $\begin{array}{l}(11,1) \\
(92) \\
(73) \\
(54)\end{array}$ & $\begin{array}{l}3^{5} \times 7^{3} \times 13 / 5 \times 11 \times 2^{25} \\
3^{3} \times 5^{2} \times 7 \times 13 / 11 \times 2^{22} \\
3^{3} \times 43^{2} / 5 \times 2^{25} \\
3^{7} / 2^{22}\end{array}$ & $\begin{aligned} 0.241371 \\
0.363468 \\
0.171834 \\
-0.227465 \\
1 / N_{(71)}=0.272\end{aligned}$ \\
\hline 10 & (84) & $\begin{array}{l}(10,2) \\
(83) \\
(64)\end{array}$ & $\begin{array}{l}3 \times 5^{2} \times 7 \times 11 / 2^{23} \\
3^{6} \times 5^{2} \times 7 \times 11 / 2^{30} \\
3 \times 5^{2} \times 7 \times 11 \times 23^{2} / 2^{30}\end{array}$ & $\begin{aligned} & 0.168712 \\
& 0.464914 \\
& 0.685958 \\
& 1 / N_{(84)}= 0.715\end{aligned}$ \\
\hline 10 & (92) & $\begin{array}{l}(12,1) \\
(10,2) \\
(83) \\
(64)\end{array}$ & $\begin{array}{l}3^{5} \times 7^{2} \times 13 / 11 \times 2^{25} \\
3 \times 5^{2} \times 7^{2} \times 13 / 2^{25} \\
3^{3} \times 5^{4} \times 7^{2} \times 13 / 11 \times 2^{30} \\
3 \times 13 \times 47^{2} / 2^{30}\end{array}$ & $\begin{aligned} 0.263357 \\
0.485254 \\
0.387962 \\
-0.115192 \\
1 / N_{(92)}=0.469\end{aligned}$ \\
\hline 12 & (88) & (84) & $\left(3^{4} \times 5^{3} \times 7^{3} / 2^{33}\right) 2.33465$ & $\begin{aligned} & 0.658502 \\
1 / N_{(\mathrm{8B})} & =0.434\end{aligned}$ \\
\hline
\end{tabular}

1) Eigenvectors and norm factors for the non-redundant cluster states are given in terms of the $c_{\left(\lambda_{\text {BMg }}\right)}$ by eqs. (47) and (48). 
where $\Psi([\ldots](\lambda \mu))$ is an abbreviated form for the cluster wave function of eq. (31), and where the normalization coefficient is given by

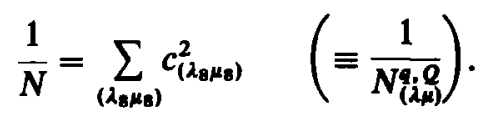

Table 4 gives the coefficients $c_{\left(\lambda_{8} \mu_{8}\right)}$ along with the normalization coefficients, $N$, for the states of greatest interest in ${ }^{20} \mathrm{Ne}$.

Under the assumption that the $A$-nucleon final state is approximated by an SU(3) strong-coupled cluster state of the form of eq. (47), the spectroscopic amplitude for the dissociation of $A$ into a ${ }^{8} \mathrm{Be}$ cluster and the residual (core) nucleus $(A-8)$ in a state $\left(\lambda_{c} \mu_{c}\right)\left[f_{c}\right] \kappa_{c} L_{c} S_{c} J_{c}$ can be denoted by

$$
A\left((A-8)_{J_{\mathrm{c}}}+{ }^{8} \mathrm{Be}\left[l \times L_{\mathrm{r}}\right]_{L_{4}} \rightarrow A_{J}\right),
$$

where $l$ is the angular momentum associated with $r_{\text {rel }} l=0,2,4$ under the assumption that the ${ }^{8} \mathrm{Be}$ internal wave function is approximated by the pure SU(3) symmetry (40); $L_{\mathrm{r}}$ is the angular momentum associated with $r_{\mathrm{c}-\mathrm{g}}$; and $L_{8}$ is the resultant in the vector coupling $\left[l \times L_{\mathrm{r}}\right] \rightarrow \boldsymbol{L}_{\mathrm{B}}$. Under the above assumptions this amplitude has the value (harmonic oscillator approximation)

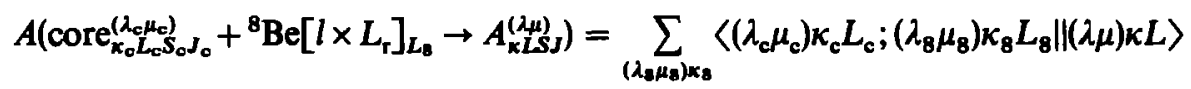

$$
\begin{aligned}
& \times\left\langle(40) l ;(Q 0) L_{\mathrm{r}} \|\left(\lambda_{8} \mu_{8}\right) \kappa_{8} L_{8}\right\rangle c_{\left(\lambda_{8} \mu_{8}\right)}(-1)^{J-J_{0}+L_{0}-L} U\left(S_{\mathrm{c}} L_{\mathrm{c}} J L_{8} ; J_{\mathrm{c}} L\right)\left(1 / N^{4=4}\left({ }^{8} \mathrm{Be}\right)\right) \text {, }
\end{aligned}
$$

where $N^{a}\left({ }^{8} \mathrm{Be}\right)$ is the normalization coefficient for the properly antisymmetrized internal wave function for ${ }^{8} \mathrm{Be}$. It has been calculated for general $q$ by Katō and Bando $\left.{ }^{17}\right)$ and has the simple value $\left[1 / N^{q}\right]^{2}=2\left[1-2^{2-\eta}\right]$, so that $1 / N^{4}\left({ }^{8} \mathrm{Be}\right)=\left[\frac{3}{2}\right]^{\frac{1}{2}}$. [In the case where the coupling $\left(\lambda_{\mathrm{c}} \mu_{\mathrm{c}}\right) \times\left(\lambda_{\mathrm{g}} \mu_{\mathrm{g}}\right) \rightarrow(\lambda \mu)$ has a multiplicity $>1$, a sum over the multiplicity label, $\rho$, must be included along with the sum over $\left(\lambda_{8} \mu_{8}\right) \kappa_{g}$; and $c_{\left(\lambda_{\left.\varepsilon, \mu_{s}\right)}\right.}$ and the first SU(3) $\supset \mathbf{R}(3)$ Wigner coefficient in (49) are then also dependent on $\rho$.]

For the eight-nucleon transfer stripping reaction on ${ }^{12} \mathrm{C}_{0^{+}}$, the ${ }^{12} \mathrm{C}_{0^{+}}+{ }^{8} \mathrm{Be} \rightarrow{ }^{20} \mathrm{Ne}$ spectroscopic amplitude is simply

$$
\begin{aligned}
& A\left({ }^{12} \mathrm{C}_{0^{+}}+\left[l \times L_{\mathrm{r}}\right]_{L_{8}=J} \rightarrow{ }^{20} \mathrm{Ne}_{\mathrm{k}}^{(\lambda \mu)}\right) \\
& \quad=\sqrt{\frac{3}{2}} \sum_{\left(\lambda_{8} \mu_{8}\right) \kappa_{8}}\left\langle(04) 0 ;\left(\lambda_{8} \mu_{8}\right) \kappa_{8} J \|(\lambda \mu) \kappa L=J\right\rangle\left\langle(40) l ;(Q 0) L_{\mathrm{r}} \|\left(\lambda_{8} \mu_{8}\right) \kappa_{8} J\right\rangle c_{\left(\lambda_{8} \mu_{8}\right)} .
\end{aligned}
$$

Even in this case there are a large number of amplitudes since all three $l$-values of 0,2 and 4 will in general contribute to an amplitude for given $L_{\mathrm{r}}$ and given final state $J$. Amplitudes for the bandheads of some of the most prominent rotational bands in ${ }^{20} \mathrm{Ne}$ are listed in table 5 by way of illustration. In a rough qualitative way the spectroscopic strength to the full rotational band structure of a given $(\lambda \mu)$ is given by the strength factors $1 / N$ listed in table 4 . 
TABLE 5

Spectroscopic amplitudes $A\left({ }^{12} \mathrm{C}_{0^{+}}+{ }^{8} \mathrm{Be}\left[l \times L_{\mathrm{r}}\right], \overrightarrow{ }^{20} \mathrm{Ne}_{k J}^{(2 \mu)}\right)$ to bandheads in some rotational bands in ${ }^{20} \mathrm{Ne}$

\begin{tabular}{|c|c|c|c|c|}
\hline$(\lambda \mu)$ & $\begin{array}{c}\text { Bandhead } \\
J^{\star}\end{array}$ & $l$ & $L_{\mathrm{r}}$ & Amplitude \\
\hline (80) & $0^{+}(\kappa=0)$ & $\begin{array}{l}0 \\
2 \\
4\end{array}$ & $\begin{array}{l}0 \\
2 \\
4\end{array}$ & $\begin{array}{r}0.3969 \\
-0.1564 \\
-0.0479\end{array}$ \\
\hline (82) & $\begin{array}{l}2^{-}(x=2) \\
4.97 \mathrm{MeV}\end{array}$ & $\begin{array}{l}2 \\
2 \\
4 \\
4\end{array}$ & $\begin{array}{l}1 \\
3 \\
3 \\
5\end{array}$ & $\begin{array}{r}-0.2957 \\
-0.2951 \\
-0.0096 \\
0.1126\end{array}$ \\
\hline (90) & $\begin{array}{l}1^{-}(\kappa=0) \\
5.80 \mathrm{MeV}\end{array}$ & $\begin{array}{l}0 \\
2 \\
2 \\
4 \\
4\end{array}$ & $\begin{array}{l}1 \\
1 \\
3 \\
3 \\
5\end{array}$ & $\begin{array}{r}0.1683 \\
-0.1744 \\
0.0251 \\
0.0933 \\
-0.1128\end{array}$ \\
\hline (42) & $\begin{array}{l}0^{+}(\kappa=0) \\
6.72 \mathrm{MeV}\end{array}$ & $\begin{array}{l}0 \\
2 \\
4\end{array}$ & $\begin{array}{l}0 \\
2 \\
4\end{array}$ & $\begin{array}{r}0.2358 \\
-0.1880 \\
0.0568\end{array}$ \\
\hline (88) & $\begin{array}{l}0^{+}(x=0) \\
7.20 \mathrm{MeV}\end{array}$ & $\begin{array}{l}0 \\
2 \\
4\end{array}$ & $\begin{array}{l}0 \\
2 \\
4\end{array}$ & $\begin{array}{r}0.3015 \\
-0.2849 \\
0.1762\end{array}$ \\
\hline
\end{tabular}

Under the assumption that the reaction $(A-8)(a, b) A$ proceeds predominantly by a direct eight-particle transfer process, the cross section for this reaction will depend on both the $\{(A-8)+8 \rightarrow A\}$ and $\{b+8 \rightarrow a\}$ amplitudes through the structure factor $B$ [see e.g. eq. (5) of ref. $\left.{ }^{60}\right)$,

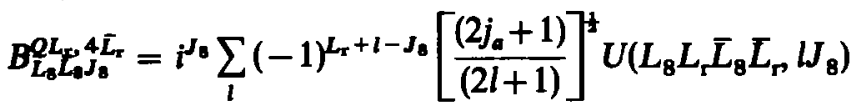

$$
\begin{aligned}
& \times A^{*}\left((A-8)_{J_{c}}+{ }^{8} \mathrm{Be}\left[l \times L_{\mathrm{r}}\right]_{L_{8}} \rightarrow A_{J}\right) A\left(b_{J_{b}}+{ }^{8} \mathrm{Be}\left[l \times L_{\mathrm{r}}\right]_{L_{\mathrm{s}}} \rightarrow a_{J_{\mathrm{a}}}\right) \text {. }
\end{aligned}
$$

For $(a, b)=\left({ }^{12} \mathrm{C}, \alpha\right)$, for example, the amplitude $A(b+8 \rightarrow a)$, in the approximation in which the internal wave functions for the ${ }^{8} \mathrm{Be}$ and $\alpha$ clusters are assumed to have pure SU(3) symmetry (40) and (00), has the simple value

$$
A\left(\alpha+{ }^{8} \mathrm{Be}\left[l \times L_{\mathrm{r}}\right]_{0} \rightarrow{ }^{12} \mathrm{C}_{0^{+}}\right)=\sqrt{\frac{3}{2}}\left\langle(40) l ;(40) L_{\mathrm{r}}=l \|(04) 0\right\rangle\left(\frac{12}{4}\right)^{2} \frac{3 \sqrt{5}}{2^{5}} a_{(04)},
$$

where $a_{(04)}$ is the amplitude of the $(\lambda \mu)=(04) L=0$ component in the ground-state wave function for ${ }^{12} \mathrm{C}$. The reduced $\mathrm{SU}(3) \supset \mathrm{R}(3)$ Wigner coefficients for $l=0$, 2 and 4 have the values $\frac{8}{15},-\left[\frac{16}{15}\right]^{\frac{1}{2}}$ and $\frac{3}{3}$, respectively. With $a_{(04)}=0.896\left[\right.$ ref. $\left.{ }^{61}\right)$, 
the amplitudes of eq. (52) are $1.11(l=0),-1.24(l=2)$ and $1.25(l=4)$ which compare with the values of $1.0830(l=0),-1.2257(l=2)$ and $1.2758(l=4)$ calculated by Kurath ${ }^{62}$ ) with more precise wave functions.

With approximations similar to the above, the $\left({ }^{14} \mathrm{~N},{ }^{6} \mathrm{Li}\right)$ amplitudes are given by

$$
\begin{aligned}
& A\left({ }^{6} \mathrm{Li}_{1^{+}}^{(20)}+{ }^{8} \mathrm{Be}\left[l \times \bar{L}_{\mathrm{r}}\right]_{L_{8}} \rightarrow{ }^{14} \mathrm{~N}_{1+}\right) \\
& \quad=\sqrt{\frac{3}{2}}\left\langle(20) 0 ;(04) L_{8}=L \|(02) L\right\rangle\left\langle(40) l ;(40) L_{\mathrm{r}} \|(04) L\right\rangle\left(\frac{14}{6}\right)^{2} \frac{15}{2^{5} \sqrt{2}} a_{L} \delta_{\bar{L}_{8} L}
\end{aligned}
$$

where $a_{0}, a_{2}$ are the coefficients of the ${ }^{13} \mathrm{~S}$ and ${ }^{13} \mathrm{D}$ [4442] components of the ${ }^{14} \mathrm{~N}$ $1^{+}$ground-state wave function. With $a_{2}=0.950, a_{0}=-0.247$ [ref. $\left.{ }^{61}\right)$ ], the $L=2$ amplitudes are (in the above approximation): $-0.221\left(l \bar{L}_{\mathrm{r}}=02\right),-0.549\left(I L_{\mathrm{r}}=22\right)$, $0.071\left(l L_{\mathrm{r}}=42\right.$ or 24$), 0.636\left(l L_{\mathrm{r}}=44\right)$; while the $L=0$ amplitudes are -0.137 $\left(l \bar{L}_{\mathrm{r}}=00\right), 0.153\left(l \bar{L}_{\mathrm{r}}=22\right),-0.154\left(l \bar{L}_{\mathrm{r}}=44\right)$.

Even for a direct eight-particle transfer process, the cross section for the $\left.{ }^{12} \mathrm{C}^{14} \mathrm{~N},{ }^{6} \mathrm{Li}\right)^{20} \mathrm{Ne}$ reaction is a function of kinematical factors for several $L_{r} \bar{L}_{\mathrm{r}}\left(L_{8} L_{8}\right) J_{8}$ combinations, so that the cross section is determined through a complicated interplay between the nuclear structure factors, $B^{Q L_{r} 4 \bar{L}_{r}}$, and kinematical factors carrying the physics of the reaction mechanism. In addition, interference with sequential two- $\alpha$-transfer processes and knock on processes may have to be considered. The former requires knowledge of $\alpha$-spectroscopic amplitudes of the type calculated in sects. 2 and 3 . The strength of the latter is determined by cluster spectroscopic amplitudes of the type $A\left({ }^{14} \mathrm{~N}+{ }^{6} \mathrm{Li} \rightarrow{ }^{20} \mathrm{Ne}\right)$ and $A\left({ }^{6} \mathrm{Li}+{ }^{6} \mathrm{Li} \rightarrow{ }^{12} \mathrm{C}\right)$ which can be calculated by the techniques used for ${ }^{8} \mathrm{Be}$ clusters in this section.

The simple SU(3), SU(4) recoupling techniques, illustrated by the calculation of some $\alpha$ and ${ }^{8} \mathrm{Be}$ cluster spectroscopic factors in this work, make it possible to calculate clustering amplitudes for any nuclear system for which SU(3) and SU(4) are meaningful symmetries. These techniques also make it possible to cope with complicated core excitations in nuclei in the $A=12-40$ region, since the properly antisymmetrized cluster wave functions are free of the spurious c.m. excitations which plague the more conventional shell model, weak coupling particle-hole model, or quartet model descriptions of such states.

It is a pleasure to acknowledge valuable discussions with A. Arima, J. P. Draayer and D. Braunschweig.

\section{Appendix}

Calculations for $\alpha$-cluster spectroscopic amplitudes require only very simple SU(3) recoupling coefficients of the form

$$
U\left((\lambda \mu)\left(Q_{1} 0\right)\left(\lambda^{\prime \prime} \mu^{\prime \prime}\right)\left(Q_{2} 0\right) ;\left(\lambda^{\prime} \mu^{\prime}\right)(Q 0)\right)
$$


with $Q=Q_{1}+Q_{2}$. An algebraic expression for these coefficients is available through the work of Biedenharn et al. [see eqs. (3.46) and (3.56) of ref. ${ }^{63}$ )]. Since the notation of ref. ${ }^{63}$ ) is very different from that used in nuclear physics applications, it may be useful to transcribe their result into the Elliott notation. The SU(3) $U$-coefficient with the "stretched" coupling $\left(Q_{1} 0\right) \times\left(Q_{2} 0\right) \rightarrow\left(Q=Q_{1}+Q_{2}, 0\right)$ is given by

$U^{2}\left((\lambda \mu)\left(Q_{1} 0\right)\left(\lambda^{\prime \prime} \mu^{\prime \prime}\right)\left(Q_{2} 0\right) ;\left(\lambda^{\prime} \mu^{\prime}\right)(Q 0)\right)$

$$
\begin{aligned}
& =\frac{Q_{1} ! Q_{2} ! \Delta_{1}^{\prime \prime} ! \Delta_{2}^{\prime \prime} ! \Delta_{3}^{\prime \prime} !\left(\lambda^{\prime}+1\right)\left(\mu^{\prime}+1\right)\left(\lambda^{\prime}+\mu^{\prime}+2\right)}{Q ! \Delta_{1} ! \Delta_{2} ! \Delta_{3} ! \Delta_{1}^{\prime} ! \Delta_{2}^{\prime} ! \Delta_{3}^{\prime} !} \\
& \times \frac{\left(\lambda+1+\Delta_{1}^{\prime \prime}\right) !\left(\lambda-\Delta_{2}\right) !\left(\lambda+\Delta_{1}-\Delta_{2}^{\prime \prime}\right) !\left(\mu+1+\Delta_{2}^{\prime \prime}\right) !\left(\mu-\Delta_{3}\right) !\left(\mu+\Delta_{2}-\Delta_{3}^{\prime \prime}\right) !}{\left(\lambda+1+\Delta_{1}\right) !\left(\lambda-\Delta_{2}^{\prime \prime}\right) !\left(\lambda+1+\Delta_{1}^{\prime \prime}-\Delta_{2}\right) !\left(\mu+1+\Delta_{2}\right) !\left(\mu-\Delta_{3}^{\prime \prime}\right) !\left(\mu+1+\Delta_{2}^{\prime \prime}-\Delta_{3}\right) !} \\
& \times \frac{\left(\lambda+\mu+2+\Delta_{1}^{\prime \prime}\right) !\left(\lambda+\mu+1-\Delta_{3}\right) !\left(\lambda+\mu+1+\Delta_{1}-\Delta_{3}^{\prime \prime}\right) !}{\left(\lambda+\mu+2+\Delta_{1}\right) !\left(\lambda+\mu+1-\Delta_{3}^{\prime \prime}\right) !\left(\lambda+\mu+2+\Delta_{1}^{\prime \prime}-\Delta_{3}\right) !}
\end{aligned}
$$

In the phase convention of ref. ${ }^{31}$ ) these $U$-coefficients are always positive. The $\Delta_{t}$ are the number of squares added to row $i$ of the Young tableau for $(\lambda \mu)$ to make the Young tableau for $\left(\lambda^{\prime} \mu^{\prime}\right)$ in the coupling $(\lambda \mu) \times\left(Q_{1} 0\right) \rightarrow\left(\lambda^{\prime} \mu^{\prime}\right)$. With $\Delta_{1}+\Delta_{2}+\Delta_{3}=Q_{1}$,

$$
\lambda^{\prime}=\lambda+\Delta_{1}-\Delta_{2}, \quad \mu^{\prime}=\mu+\Delta_{2}-\Delta_{3}^{\prime} .
$$

Similarly, $\Delta_{i}^{\prime}$ are the number of squares added to row $i$ of the täbleau for $\left(\lambda^{\prime} \mu^{\prime}\right)$ to make the tableau for $\left(\lambda^{\prime \prime} \mu^{\prime \prime}\right)$ in the coupling $\left(\lambda^{\prime} \mu^{\prime}\right) \times\left(Q_{2} 0\right) \rightarrow\left(\lambda^{\prime \prime} \mu^{\prime \prime}\right)$; with $\Delta_{1}^{\prime}+\Delta_{2}^{\prime}+\Delta_{3}^{\prime}=Q_{2}$. Finally, $\Delta_{i}^{\prime \prime}=\Delta_{i}+\Delta_{i}^{\prime}$, so that

$$
\lambda^{\prime \prime}=\lambda^{\prime}+\Delta_{1}^{\prime}-\Delta_{2}^{\prime}=\lambda+\Delta_{1}^{\prime \prime}-\Delta_{2}^{\prime \prime}, \quad \mu^{\prime \prime}=\mu^{\prime}+\Delta_{2}^{\prime}-\Delta_{3}^{\prime}=\mu+\Delta_{2}^{\prime \prime}-\Delta_{3}^{\prime \prime} .
$$

\section{References}

1) D. A. Goldberg, J. B. Marion and S. J. Wallace, Clustering phenomena in nuclei II, College Park, Maryland (1975)

2) $\mathbf{K}$. Wildermuth and W. McClure, Cluster representations of nuclei, Springer Tracts in Modern Physics, vol. 41 (Springer, Berlin, 1966)

3) A. Arima, H. Horiuchi, K. Kubodera and N. Takigawa, Advances in nuclear physics, vol. 5, ed. M. Baranger and E. Vogt (Plenum Press, NY, 1973)

4) M. Ichimura, A. Arima, E. C. Halbert and T. Terasawa, Nucl. Phys. A204 (1973) 225

5) H. H. Hackenbroich, Z. Phys. 231 (1970) 216;

P. Heiss and H. H. Hackenbroich, Z. Phys. 235 (1970) 422;

H. H. Hackenbroich, T. H. Seligman and W. Zahn, Ann. of Phys., to be published

6) H. Friedrich, Nucl. Phys. A224 (1974) 537

7) T. H. Seligman and W. Zahn, J. of Phys. G2 (1976) 79

8) W. Sunkel and K. Wildermuth, Phys. Lett. 41B (1972) 439

9) P. Kramer and D. Schenzle, Rev. Mex. Fiz. 22 (1973) 25

10) H. Hutzelmeyer and H. H. Hackenbroich, Z. Phys. 232 (1970) 356

11) H. H. Hackenbroich, T. H. Seligman and W. Zahn, Nucl. Phys. A2s9 (1976) 445

12) H. H. Hackenbroich, T. H. Seligman, W. Zahn and D. Fick, Phys. Lett. 62B (1976) 121

13) M. LeMere, Y. C. Tang and D. R. Thompeon, Phys. Rev. C14 (1976) 23; Nucl. Phys. A266 (1976) 1

14) H. Horiuchi and Y. Suzuki, Prog. Theor. Phys. 4 (1973) 1974

15) Y. Suzuki, Prog. Theor. Phys. 50 (1973) 341 
16) H. Horiuchi, Prog. Theor. Phys. 51 (1974) 745

17) K. Kato and H. Bando, Prog. Theor. Phys. 53 (1975) 692

18) M. Kamimura and T. Matsuse, Prog. Theor. Phys. 51 (1974) 438

19) S. Saito, Prog. Theor. Phys. 41 (1969) 705

20) Y. Suzuki, Prog. Theor. Phys. 55 (1976) 1751; 56 (1976) 111

21) T. Matsuse and M. Kamimura, Prog. Theor. Phys. 49 (1973) 1765

22) F. Nemoto, Y. Yamamoto, H. Horiuchi, Y. Suzuki and K. Ikeda, Prog. Theor. Phys. S4 (1975) 104

23) H. Bandō, Nucl. Phys. A261 (1976) 269

24) T. Tomoda and A. Arima, Proc. of Int. Symp. on cluster structure of nuclei and transfer reactions induced by heavy ions, Tokyo (1975)

25) H. Horiuchi, Prog. Theor. Phys. 51 (1974) 1266; 53 (1975) 447

26) P. Kramer and D. Schenzle, Nucl. Phys. A204 (1973) 593

27) P. Kramer and T. H. Seligman, Nucl. Phys. A136 (1969) 545; A186 (1972) 49

28) P. Kramer and D. Schenzle, to be published as quoted in ref. ')

29) I. V. Kurdyumov, Yu. F. Smirnov, K. B. Shitikove and S. El. Samarai, Nucl. Phys. A145 (1970) 593

30) V. P. Korennoy, I. V. Kurdyumov, V. G. Neudatchin and Yu. F. Smirnov, Phys. Lett. 4 B (1972) 607; Yad. Fiz. 17 (1973) 750

31) J. P. Draayer and Y. Akiyama, J. Math. Phys. 14 (1973) 1904

32) Y. Akiyama and J. P. Draeyer, Comp. Phys. Commun. 5 (1973) 405

33) K. T. Hecht and S. C. Pang, J. Math. Phys. 10 (1969) 1571

34) K. T. Hecht and D. Braunschweig, Nucl. Phys. A244 (1975) 365

35) J. P. Draayer, Nucl. Phys. A237 (1975) 157

36) N. Anantaraman, C. L. Bennett, J. P. Draayer, H. W. Fulbright, H. E. Gove and J. Tōke, Phys. Rev. Lett. 35 (1975) 1131

37) N. Anantaraman, H. E. Gove, J. Tōke and J. P. Draayer, Phys. Lett. 60B (1976) 149;

J. P. Drasyer, H. E. Gove, J. P. Trentelman, N. Anantaraman and R. M. DeVries, Phys. Lett. 53B (1974) 250

38) N. Anantaraman, H. E. Gove, J. Toke and J. P. Draayer, Nucl. Phys., to be published

39) J. D. Cossairt, R. D. Bent, A. S. Broad, F. D. Becchetti and J. Janecke, Nucl. Phys. A261 (1976) 373;

A. M. Van der Molen, J. Janecke and F. D. Becchetti, p.413 of ref. ${ }^{1}$ )

40) M. Conze, Ph.D. Dissertation, Technische Hochschule Darmstadt (1976)

41) A. Arima, H. Horiuchi and T. Sebe, Phys. Lett. 24B (1967) 129

42) P. J. Ellis and T. Engeland, Nucl. Phys. A144 (1970) 161 ; A181 (1972) 368; Phys. Lett. 25B (1967) 57

43) R. Middleton, J. D. Garrett and H. T. Fortune, Phys. Rev. Lett. 27 (1971) 950; 27 (1971) 129

44) K. Nagatani, M. J. LeVine, T. A. Belote and A. Arima, Phys. Rev. Lett. 27 (1971) 1071

45) N. Marquardt, W. von Oertzen and R. L. Walter, Phys. Lett. $35 B$ (1971) 37

46) R. Middleton, J. D. Garrett and H. T. Fortume, Phys. Lett. 39B (1972) 339

47) H. T. Fortune, R. R. Betts, J. N. Bishop, M. N. I. Al-Jadir and R. Middleton, Phys. Lett. 55B (1975) 439

48) P. Braun-Munzinger, C. K. Gelbke, N. Grama, H. Homeyer, E. Ridinger and R. Stock, Phys. Rev. Lett. 29 (1972) 1261

49) H. Feshbach, Topics in the theory of nuclear reactions; Documents on Modern Physics (Gordon and Breach, NY, 1973)

50) M. N. Aljadir, Nucl. Phys. A251 (1975) 156

51) P. Kramer, Rev. Mex. Fis. 19 (1970) 241

52) P. Kramer, Z. Phys. 205 (1967) 181

53) P. Kramer, Z. Phys. 216 (1968) 68

54) K. T. Hecht, Bull. Am. Phys. Soc. 21 (1976) 614; and to be published

55) Y. Akiyama, A. Arima and T. Sebe, Nucl. Phys. A138 (1969) 273

56) T. A. Brody and M. Moshinsky, Rev. Mex. Fis. 9 (1960) 181

57) B. Kaufman and C. Noack, J. Math. Phys. 6 (1965) 142

58) A. Arima, V. Gillet and J. Ginocchio, Phys. Rev. Lett. 25 (1970) 1043

59) M. Harvey, Nucl. Phys. A202 (1973) 191

60) D. Kurath and I. S. Towner, Nucl. Phys. A222 (1974) 1

61) A. N. Boyarkina, Izv. Akad. Nauk USSR 23 (1964) 337 (English Transl., p. 255)

62) D. Kurath, Phys. Rev. C7 (1973) 1390

63) L. C. Biedenharn, J. D. Louck, E. Chacon and M. Ciftan, J. Math. Phys. 13 (1972) 1957 